\title{
REVIEWS
}

\section{Of ticks, mice and men: understanding the dual-host lifestyle of Lyme disease spirochaetes}

\author{
Justin D. Radolf ${ }^{1,2}$, Melissa J. Caimano ${ }^{1}$, Brian Stevenson ${ }^{3}$ and Linden T. Hu
}

Abstract | In little more than 30 years, Lyme disease, which is caused by the spirochaete

Borrelia burgdorferi, has risen from relative obscurity to become a global public health

problem and a prototype of an emerging infection. During this period, there has been an

extraordinary accumulation of knowledge on the phylogenetic diversity, molecular biology,

genetics and host interactions of B. burgdorferi. In this Review, we integrate this large body of

information into a cohesive picture of the molecular and cellular events that transpire as

Lyme disease spirochaetes transit between their arthropod and vertebrate hosts during the

enzootic cycle.

\section{Oligoarthritis}

Arthritis affecting more than one joint in an asymmetrical pattern.

Pathognomonic Characteristic for a particular disease.

${ }^{1}$ Department of Medicine and Department of Pediatrics, University of Connecticut Health Center.

${ }^{2}$ Department of Genetics and Developmental Biology and Department of Immunology, University of Connecticut Health Center, Farmington, Connecticut 06030, USA.

${ }^{3}$ Department of Microbiology, Immunology, and Molecular Genetics, University of Kentucky College of Medicine, Lexington, Kentucky 40536,

USA.

${ }^{4}$ Division of Geographic Medicine and Infectious Diseases, Tufts Medical Center, Boston,

Massachusetts 02067, USA

Correspondence to J.D.R. e-mail: JRadolf@up.uchc.edu doi:10.1038/nrmicro2714 Published online

9 January 2012
Since its original description, Lyme disease (Lyme borreliosis) has risen from relative obscurity to become a prototypical emerging infectious disease ${ }^{1}$. The path to notoriety began with a little noticed epidemic of oligoarthritis in the mid 1970s, mainly in children, in several rural communities clustered about the town of Lyme in southeastern Connecticut, USA ${ }^{2}$. Physicians misdiagnosed many of these children as having juvenile rheumatoid arthritis, leading two astute mothers to seek the assistance of investigators at nearby Yale University (New Haven, Connecticut) ${ }^{1}$. The observation that approximately one-quarter of patients developed an expanding, annular skin lesion before the onset of arthritis proved the key to solving the medical mystery. The pathognomonic 'bull's eye' rash, which is now called erythema migrans (FIG. 1), had been closely associated with the bite of the sheep tick (castor bean tick) Ixodes ricinus in Northern Europe and was widely believed to be caused by an unknown infectious agent, possibly a spirochaete ${ }^{1}$. These early field investigations prompted an intensive hunt that led to the isolation of the causative organism from a species of North American deer tick, Ixodes scapularis ${ }^{3}$. Shortly thereafter, a novel spirochaete was isolated from skin, blood and cerebrospinal fluid specimens obtained from patients with erythema migrans $s^{4,5}$. DNA-DNA hybridization studies revealed the isolate to be a new species within the genus Borrelia ${ }^{6}$, and the species was subsequently named Borrelia burgdorferi after its co-discoverer, Willy Burgdorfer.

Lyme disease spirochaetes are widely distributed throughout the temperate zones of the Northern
Hemisphere, and their range continues to expand as lands that were denuded for agriculture become reforested, creating new habitats for deer, ticks and infection-susceptible vertebrates ${ }^{7}$. Lyme disease accounts for $>90 \%$ of all vector-borne disease in the United States, with nearly 30,000 confirmed cases reported in 2008 (REF. 8). As many as 60,000 cases occur each year within the range of $I$. ricinus, the primary vector in Europe 9 .

The expanding universe of Borrelia burgdorferi Borrelia spp. fall into two major phyletic groups, one containing the causative agents of Lyme disease and the other containing the spirochaetes responsible for relapsing fever ${ }^{7}$. Phylogenetic analyses have led to the division of Lyme disease spirochaetes into numerous species, collectively referred to as B. burgdorferi sensu lato (s.l.). Among the more than 20 named and unnamed species in the B. burgdorferi s.l. complex, three genospecies predominate as human pathogens: $B$. burgdorferi sensu stricto (s.s.) in the United States and Western Europe, and Borrelia garinii and Borrelia afzelii in Eurasia ${ }^{7}$. Four other species, Borrelia valaisiana, Borrelia lusitaniae, Borrelia spielmanii and Borrelia bissettii, have also been isolated or detected by PCR in specimens from small numbers of patients ${ }^{7}$. Hereafter, B. burgdorferi is used to refer to B. burgdorferi s.l.

\section{The enzootic cycle}

The life cycle of Lyme disease spirochaetes is depicted in FIG. 1.B. burgdorferi is transmitted principally by four species of hard tick within the I. ricinus complex: 


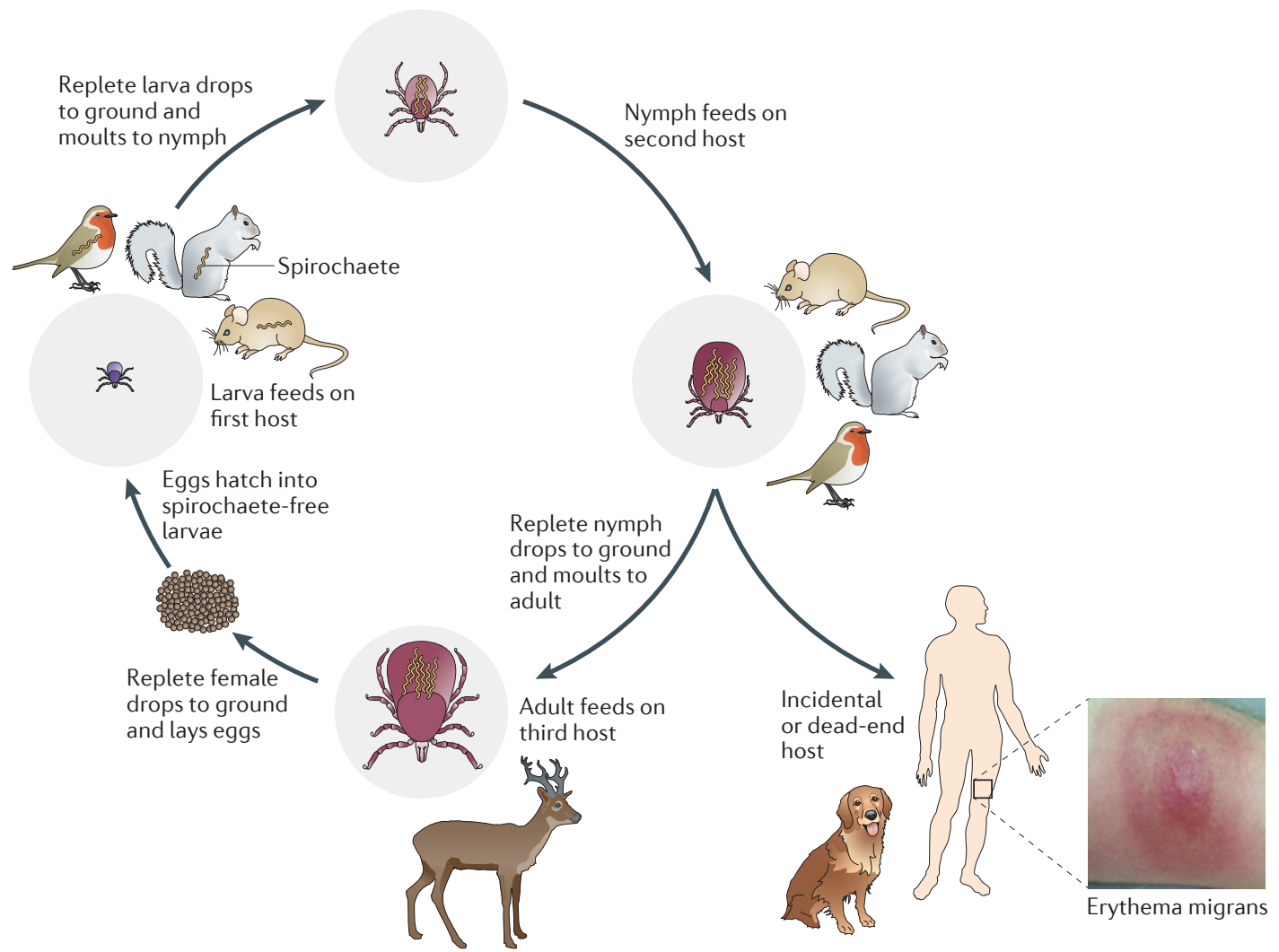

Spirochaete

A member of the ancient and deeply branching bacterial phylum Spirochaetes, which consists of bacteria that possess a helically coiled (spiral-shaped) or wave-like morphology and a distinctive mode of motility that enables them to penetrate viscous media and tissues.

\section{Genospecies}

A term often used to describe different species of Lyme disease spirochaetes that tend to occur in particular geographic regions of the Northern Hemisphere.

Transovarial

Passed from a female adult to the larva via the egg (as occurs with spirochaetes that cause relapsing fever but not those that cause Lyme disease).

\section{Reservoir}

In the context of Lyme disease, a vertebrate species that can be persistently and asymptomatically infected with spirochaetes and, therefore, can serve as a source of infection for naive feeding ticks, usually larvae.

\section{Trans-stadial}

Transmitted to successive developmental stages of the tick.

\section{Enzootic}

Existing in nature in animal reservoirs.

Figure 1 | The enzootic cycle of Borrelia burgdorferi. Ixodes spp. ticks undergo a three-stage life cycle — larva, nymph and adult - with one blood meal per stage. Although some Borrelia spp. that cause relapsing fever can be passed from adult to egg (transovarial transmission), this does not occur with Borrelia burgdorferi sensu lato, so each generation of tick must acquire a B. burgdorferi infection anew. Larval ticks feed on many different animals, including Peromyscus spp. mice, squirrels and birds. B. burgdorferi infection is acquired by feeding on an infected reservoir animal, and the bacterium is retained during the subsequent stages (that is, trans-stadially) after each blood meal and moult ${ }^{7,10}$. Nymphs feed on a similar range of hosts to larvae; transmission of spirochaetes to a competent reservoir host by a feeding nymph perpetuates the enzootic cycle for the next generation of larval ticks. Although small mammals are usually thought of as the primary reservoirs for Lyme disease spirochaetes, studies have called attention to the importance of migratory birds as disseminators of spirochaetes over large distances ${ }^{7,10}$. Adult ticks are not generally important for maintenance of $B$. burgdorferi in the wild, as they feed predominantly on larger animals such as deer, which are incompetent hosts for B. burgdorferi ${ }^{7}$. However, deer are important for maintenance of the tick population because adult ticks mate on them. Although all three stages of Ixodes spp. can feed on humans, nymphs are responsible for the vast majority of spirochaete transmission to humans. It is unknown whether infected humans can transmit spirochaetes to feeding larvae, and humans are generally considered dead-end hosts. Dogs are probably incidental hosts and not part of the enzootic cycle.

I. scapularis and Ixodes pacificus (the western blacklegged tick) in eastern and western North America, respectively, I. ricinus in Europe and Ixodes persulcatus (the tiaga tick) in Asia ${ }^{7,10}$. The tick larvae are uninfected when they hatch, as there is no transovarial transmission, and B. burgdorferi is acquired after feeding on an infected reservoir host. After moulting to the nymphal stage, the ticks transmit the pathogen to the animal that provides its next blood meal. Larvae of hard ticks other than Ixodes spp. inefficiently acquire Lyme disease spirochaetes from a blood meal and/or fail to maintain them through the trans-stadial moult ${ }^{11}$. The Ixodes spp. that transmit B. burgdorferi to humans tend to feed on diverse species of vertebrates, accounting for the geographical breadth of transmission cycles within the Northern Hemisphere. Their generalist feeding behaviour is also responsible for the incidental infection of humans ${ }^{7,10}$. Field studies in North America and Eurasia have identified a variety of small-mammal and avian reservoirs in enzootic transmission cycles ${ }^{7}$. The whitefooted mouse, Peromyscus leucopus, is considered to be the main reservoir in the northeastern United States, whereas rodents and migratory birds are the principal reservoirs in Europe for B. afzelii and B. garinii, respectively.

\section{Outcomes of infection}

Spirochaetes are deposited into the bite wound along with the tick saliva during tick feeding ${ }^{12}$. Infection rarely, if ever, occurs during the first 24 hours of nymphal feeding but becomes increasingly likely after the tick has been attached for 48 hours or longer ${ }^{13}$. The outcome of the inoculating event depends on the recipient. White-footed mice remain persistently infected without 


\section{Box 1 | Animal models of Lyme disease}

One of the important early advances in the study of Lyme disease was the development of animal models of infection. As mice and other rodents are natural reservoirs for Borrelia burgdorferi sensu lato, the causative agent, it is not surprising that wild Peromyscus spp. mice show no observable changes during infection with B. burgdorferi, as symptomatic disease could leave the infected animal with a survival disadvantage and/or limit the opportunities for transmission of spirochaetes to new cohorts of vectors ${ }^{128}$. However, when infected with $B$. burgdorferi, specific inbred strains of the laboratory mouse Mus musculus have been found to exhibit features similar to those of human Lyme disease ${ }^{14,15}$. For example, $\mathrm{C} 3 \mathrm{H}$ and BALB/c mice develop both ankle joint arthritis and carditis on infection with B. burgdorferi, whereas C57BL/6 and DBA mice are more resistant to developing signs of infection and typically have only minimal inflammation in the heart and joints. The greater disease susceptibility of $\mathrm{C} 3 \mathrm{H}$ mice does not seem to be due to greater control of the infection, as the spirochaete numbers are comparable to those in disease-resistant mouse strains. By contrast, in BALB/c mice, spirochaete numbers and signs of inflammation directly correlate with the inoculum size, suggesting that the mechanisms of arthritis and carditis are different in this mouse strain. No strain of inbred mouse develops erythema migrans, meningitis or encephalitis, and thus all are imperfect models for human Lyme disease. Also unknown is whether the genetic determinants controlling the development of arthritis and carditis in inbred mice are related to those responsible for the corresponding manifestations in humans.

Other animals have been used to model specific features of human Lyme disease. Dogs infected with B. burgdorferi can develop arthritis and facial nerve palsies ${ }^{129}$. Rhesus monkeys can also be infected with B. burgdorferi and are used as a model of neuroborreliosis because of their propensity for central nervous system infection, particularly when immunosuppressed with corticosteroids ${ }^{14}$. Rhesus monkeys also develop erythema migrans, mononeuritis multiplex and arthritis, making them the animal model that is most similar to humans for this disease ${ }^{14}$. However, for reasons of cost and ease of genetic manipulation, animal models other than mice have been studied sparingly.

\author{
Carditis \\ Inflammation of the heart: \\ Lyme carditis is caused by \\ spirochaete infection of the \\ heart. \\ Spirochaetaemia \\ Dissemination of spirochaetes \\ through the bloodstream.

\section{Lipoprotein} \\ A protein containing covalently \\ bound fatty acids that, in \\ bacteria, are typically at the \\ amino terminus.

\section{Auxotroph} \\ An organism that is unable \\ to synthesize an essential \\ nutrient.

\section{Dps} \\ An oligomeric, ferritin-like \\ protein that protects DNA \\ against damage meditated by \\ oxidative stress and starvation.
}

evidence of inflammation ${ }^{14}$. Although all laboratory strains of inbred mice are susceptible to infection, the development of organ system pathology (that is, arthritis and carditis) in immunocompetent mice is highly strain dependent ${ }^{14,15}$ (BOX 1).

The B. burgdorferi genome does not encode any known toxins or the machinery that would be required to secrete them ${ }^{16}$; tissue damage, and hence disease, is believed to be mediated by the inflammatory response elicited in the mammalian host ${ }^{15,17}$. Although the natural history of Lyme disease in humans is variable and poorly defined ${ }^{17}$, seroprevalence surveys in endemic areas have revealed that asymptomatic or subclinical infections occur frequently ${ }^{18}$. Erythema migrans, the most common clinical manifestation of borrelial infection, develops following an incubation period of 3-32 days ${ }^{17}$. Low-level spirochaetaemia probably occurs in the majority of untreated patients ${ }^{19}$, occasionally affecting the peripheral or central nervous system, joints or heart ${ }^{17}$. In Europe, most dermatological manifestations are attributed to $B$. afzelii, and neuroborreliosis is caused mostly by $B$. garinii ${ }^{20}$. The relative infrequency of B. burgdorferi s.s. as a cause of disease in Europe is believed to explain why carditis and arthritis are less common sequelae of infection here than in North America $^{20}$. Differences in infectivity have also been noted between isolates of B. burgdorferi s.s. ${ }^{21,22}$.

The three decades since the discovery of B. burgdorferi have witnessed an impressive accumulation of knowledge on the molecular biology of this pathogen, its interactions with its arthropod vector, its virulence determinants, the immune responses it elicits during mammalian infection and the mechanisms involved in immune evasion. In the remainder of this Review, we integrate this knowledge into a cohesive picture of the molecular and cellular events that sustain the B. burgdorferi enzootic cycle.

\section{Genomics and cellular architecture}

Genomic complexity coupled with metabolic parsimony. Members of the genus Borrelia possess the most complex genomes of all known bacteria ${ }^{16,23}$. More than 20 distinct, naturally occurring genetic elements have been identified in the B31 type strain of B. burgdorferi. These include the $\sim 1 \mathrm{Mb}$ linear chromosome and an assortment of linear and circular plasmids totalling approximately $600 \mathrm{~kb}^{16,23}$. The chromosome carries the majority of housekeeping genes and is fairly constant in organization and content across the genus ${ }^{23}$. The plasmids, which encode most of the differentially expressed outer-surface lipoproteins, exhibit much greater variability in gene content, are not equally represented in all strains and, in laboratory models, are not all essential for maintenance of the enzootic cycle ${ }^{23}$. One of the most curious features of the borrelial genome is its degree of redundancy. This is reflected not only in the extraordinarily large number of paralogous gene families distributed mainly among its plasmid complement, but also in the cp32 plasmid family, members of which contain large stretches of essentially identical DNA interrupted by sequence-variable lipoprotein genes ${ }^{23}$. TABLE 1 summarizes the key genes that are currently believed to contribute to maintenance of the B. burgdorferi enzootic cycle.

The metabolic abilities of $B$. burgdorferi are extremely limited as a result of reductive evolution following the adoption of a lifestyle that involves parasitizing nutrients from its hosts ${ }^{16,24}$. The bacterium is an auxotroph for all amino acids, nucleotides and fatty acids. It also lacks genes encoding enzymes for the tricarboxylic acid cycle and oxidative phosphorylation, deriving energy instead from the fermentation of sugars to lactic acid via the Embden-Meyerhof pathway ${ }^{24,25}$. One of the metabolic oddities of B. burgdorferi is that it does not seem to require iron. Spirochaetes grown in vitro have no measurable iron content ${ }^{26}$ and, with the possible exception of BB0690, a Dps-like bacterioferritin orthologue ${ }^{27}$ (formerly known as NapA), the genome does not encode orthologues for any known iron-requiring metalloproteins, such as cytochromes, catalase or superoxide dismutase ${ }^{16,26}$.

Cell envelope. The structure, composition and physical properties of the cell envelope of $B$. burgdorferi diverge markedly from those of prototypical proteobacteria such as Escherichia coli ${ }^{28}$ (FICS 2,3). The outer membrane is a fluid and fragile bilayer that is devoid of lipopolysaccharide ${ }^{16,29}$. Its major lipid constituents are phosphatidylcholine, phosphatidylglycerol and the highly immunogenic but non-inflammatory glycolipids cholesteryl-6-O-acyl- $\beta$-D-galactopyranoside and cholesteryl- $\beta$-D-galactopyranoside ${ }^{30}$, none of which is 
Table 1 | Key genes thought to contribute to maintenance of the Borrelia burgdorferi sensu lato enzootic cycle

Gene*

Name and/or function of encoded protein

References

Chromosome

glp operon (BB_0240-BB_0243)

- Glycerol utilization within ticks

57,69

bb0365

- Persistence in ticks

bmpA and bmpB (BB_0383 and BB_0382)

- Laminin binding

bb0690

- Establishment of joint infection

cdr (BB_0728)

- Dps-like bacterioferritin orthologue

- Persistence in ticks

hk1 and rrp1 (BB_0420 and BB_0419)

- Coenzyme A disulfide reductase

- Histidine kinase 1 and response regulatory protein 1, respectively

plzA (BB_0733)

- PilZ domain-containing cyclic di-GMP effector protein

pdeA (BB_0363)

- Required for infection of ticks and mice

- Required to establish infection in mice

pdeB (BB_0374)

- Phosphodiesterase B (HD-GYP-type phosphodiesterase)

p66 (BB_0603)

- Required for survival in ticks and for tick-to-mammal transmission

- Integrin-binding adhesin

bosR (BB_0647)

- Borrelia oxidative stress regulator

csrA (BB_0184)

- Fur-PerR orthologue required for transcription of rpoS

rrp2 (BB_0763)

- RNA-binding protein involved in post-transcriptional regulation of rpoS

$42-45$

- Response regulator protein 2

- Required by RpoN for transcription of rpoS

rpoN (BB_0450)

- Alternative $\sigma$-factor that transcribes rpoS

- Alternative $\sigma$-factor that transcribes mammalian-phase genes and represses tick-phase genes

hrpA (BB_0827)

- DEAH-box RNA helicase involved in global gene regulation

resT (BB_B03)

- Telomere resolvase

- Dissemination within mice

guaA and guaB (BB_B18 and BB_B17)

- GMP synthase and inosine monophosphate dehydrogenase, respectively

- Involved in the purine salvage pathway

\section{cp32 family plasmids}

ospE alleles such as erpP (BB_N38), erpC (GenBank acession AAC34910.1) and erpA (BB_L39 and BB_P38)

\section{Plasmid lp25}

bbe02

bptA (BB_E16)

pncA (BB_E22)

\section{Plasmid lp28-1}

vls locus

- BbCRASP3, BbCRASP4 and BbCRASP5, respectively

- Complement inhibition

- Laminin binding

- Plasminogen binding

$81-83,106$ 110,111 ,

141,142

- Restriction-modification system

- Persistence in ticks

- Pyrazinaminidase-nicotinamidase

- Recombinatorial system for immune evasion

- BbCRASP2

cspZ (BB_H06)

- Complement inhibition

Plasmid lp 36

ade (BB_K17)

- Adenine deaminase

- Fibronectin-binding protein 
Table 1 (cont.) | Key genes thought to contribute to maintenance of the Borrelia burgdorferi sensu lato enzootic cycle

\begin{tabular}{|c|c|c|}
\hline Gene* & Name and/or function of encoded protein & References \\
\hline \multicolumn{3}{|l|}{ Plasmid lp54 } \\
\hline ospA and ospB (BB_A15 and BB_A16) & - Colonization of tick midgut epithelium & $65-67$ \\
\hline$b b a 52$ & - Tick-to-mammal transmission & 151 \\
\hline lp6.6 (BB_A62) & $\begin{array}{l}\text { - Lipoprotein } 6.6 \\
\text { - Persistence in ticks }\end{array}$ & 152 \\
\hline $\operatorname{csp} A$ (BB_A68) & $\begin{array}{l}\text { - BbCRASP1 } \\
\text { - Outer-surface protein } \\
\text { - Complement inhibition }\end{array}$ & $84,154,155$ \\
\hline
\end{tabular}

BbCRASP, complement regulator-acquiring surface protein. *Locus identifiers are provided in brackets.

found in the typical proteobacterial outer membrane. The cholesterol glycolipids form raft-like microdomains that are required for the complement-independent bactericidal activity of antibodies directed against lipoproteins on the outer surface of the spirochaete ${ }^{29}$. The borrelial outer membrane also contains a low density of proteins with membrane-spanning domains ${ }^{28}$. Some of these proteins possess channel-forming properties, although they lack sequence similarity to the better studied bacterial porins ${ }^{28}$. BesC, a TolC orthologue encoded by BB_0142, forms part of an efflux system that is required for virulence and antimicrobial resistance ${ }^{31}$.

From the standpoint of pathogenesis, the most notable difference between the outer membranes of $B$. burgdorferi and proteobacteria is the number and variety of lipoproteins that adorn the spirochaete surface ${ }^{28}$ (FICS 2,3). Characterization of differentially expressed outer-surface lipoproteins has been a major focus of research, including vaccine development. How Lyme disease spirochaetes direct nascent lipoproteins to their surface is one of the mysteries of borrelial cell biology. B. burgdorferi contains a lipoprotein outer-membrane localization (LOL) system similar to that of proteobacteria for directing newly exported lipoproteins to the outer membrane, although the borrelial LOL system uses entirely different sorting rules to the E. coli prototype $^{28}$. However, B. burgdorferi lacks any of the known pathways for translocating lipoproteins across the outer membrane to the outer surface ${ }^{28}$. A $\beta$-barrel assembly machinery A (BamA) orthologue ${ }^{32}$ seems to be the central component of the $B$. burgdorferi outer-membrane assembly machinery. The outer membranes of BamAdepleted spirochaetes are deficient in outer-surface lipoproteins as well as membrane-spanning $\beta$-barrels, suggesting that an as-yet-unidentified integral outermembrane protein is involved in directing lipoproteins to the spirochaete surface.

Motility. B. burgdorferi moves by posteriorly propagating planar waves ${ }^{33}$. A defining morphological feature of B. burgdorferi, as with all spirochaetes, is that the organelles of motility - the flagella - are contained entirely within the periplasmic space ${ }^{33}$ (FIG. 2d). The filaments are attached at each cell pole to linearly arranged flagellar motors (7-11 at each end), which are elaborate nanomachines that convert chemiosmotic potential into motive force ${ }^{34}$. Cryoelectron tomography has revealed that the flagellar filaments form flat ribbons that wind along the cell cylinder on top of the peptidoglycan layer and below the outer membrane ${ }^{35}$ (FIG. 2d). Parallel and adjacent to the motors are arrays of methyl-accepting chemotaxis proteins ${ }^{36}$ (FIG. 2b) that bind attractant and repellent molecules through their periplasmic domains, transmitting environmental signals to the motors by modulating the phosphorylation state of the chemotaxis (Che) two-component system, CheA-CheY ${ }^{33}$.

\section{Regulation of differential gene expression}

Considering the extensive transcriptional changes that the spirochaete undergoes throughout the enzootic cycle, the apparatus for controlling gene expression seems surprisingly sparse. However, it is becoming increasingly apparent that layers of regulatory complexity do in fact exist ${ }^{37,38}$. Many genes that encode proteins required for transmission of spirochaetes between vector and vertebrate hosts are transcribed by an alternative RNA polymerase $\sigma$-factor, RpoS, which is transcribed by another alternative $\sigma$-factor, RpoN (also known as $\sigma^{54}$ and NtrA $)^{37-41}$ (FIG. 4a). RpoN-dependent transcription of $r p o S$ requires the formation of an open $r p o S$ promoter complex, mediated by phosphorylated response regulatory protein $2(\mathrm{Rrp} 2)$ and presumed metal-dependent DNA binding by a Fur-PerR orthologue, Borrelia oxidative stress regulator (BosR; also known as Fur), upstream of the rpoS promoter ${ }^{37,38,42-47}$ (FIG. 4a). The phosphate group for Rrp2 phosphorylation seems to come from acetyl phosphate, the intermediate of the acetate kinasephosphate acetyltransferase pathway that converts acetate to acetyl-CoA ${ }^{48}$ (FIG. 4a). In addition, rpoS expression is post-transcriptionally regulated by the small RNA $\mathrm{DsrA}^{49}$, the RNA chaperone $\mathrm{Hfq}^{50}$ and the RNA-binding protein CsrA $^{51,52}$.

Two DNA-binding proteins - BpaB (encoded by multiple alleles per isolate) and EbfC - are involved in regulation of the cp32-encoded ospE, ospF and elp lipoprotein gene families (also collectively known as erp 


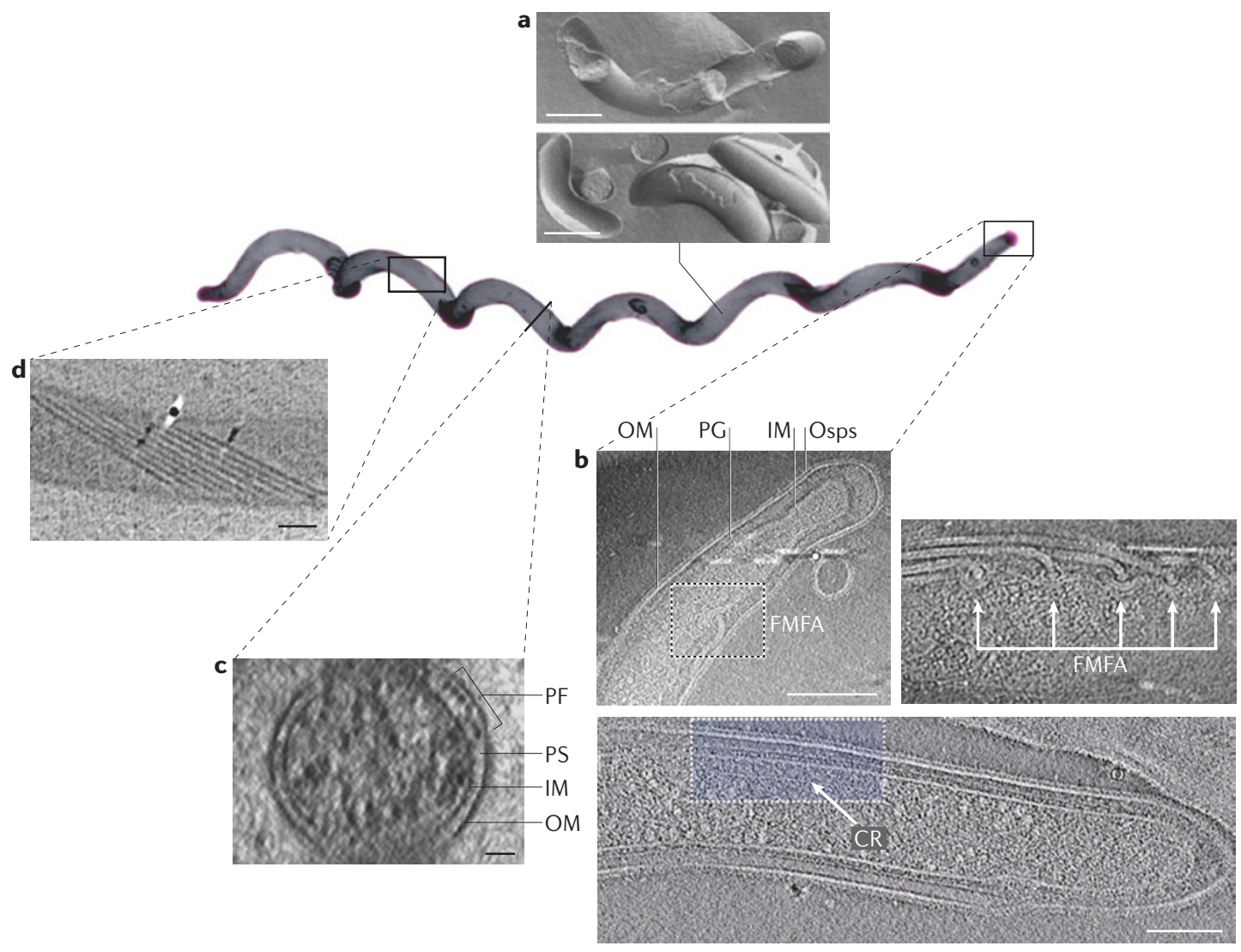

Figure 2 | Cellular architecture of Borrelia burgdorferi. a | Freeze-fracture electron micrographs showing the convex and concave leaflets of the inner and outer membranes (IM and OM, respectively). Integral membrane proteins (particles) are considerably more abundant in the IM; the density of OM particles is much lower than that in a prototypical Gram-negative bacterium. Scale bars represent $500 \mathrm{~nm}$. b|Cryoelectron tomograms of the ends of borrelial cells, showing IM, OM, peptidoglycan (PG), flagellar motor and filament assemblies (FMFA), chemoreceptor arrays (CR) and an external layer comprising outer-surface lipoproteins (Osps). Upper scale bar represents $1 \mu \mathrm{m}$, lower scale bar represents $100 \mathrm{~nm}$. c |Cryoelectron tomographic cross-section showing the IM, OM, periplasmic space (PS) and periplasmic flagella (PFs). Scale bar represents $50 \mathrm{~nm}$. d | Longitudinal cryoelectron tomographic slice showing a ribbon of nine flagellar filaments wrapping around the IM in a right-handed helix. Scale bar represents $200 \mathrm{~nm}$. Part a is reproduced, with permission, from REF. 130 (C) (1994) American Society for Microbiology (ASM). Part b is reproduced, with permission, from REF. 34 (C) (2009) ASM, and from REF. 36 (c) (2011) ASM. Parts c,d are reproduced, with permission, from REF. 35 ( (2009) ASM.

Hypostome

A barbed protuberance of the mouthparts that anchors the tick during the blood meal. genes $)^{53}$. EbfC is also involved in regulating the expression of other borrelial genes, including those encoding components of the oligopeptide permease $\mathrm{ABC}$ transporter ${ }^{54}$. The DNA-binding protein $\mathrm{Hbb}$ has a role in regulating the expression of the gene encoding p66, a porin and adhesin $^{55}$. As a final example, a two-component system composed of histidine kinase 1 (Hk1) and Rrp1 controls production of the second messenger molecule cyclic di-GMP (c-di-GMP) in response to stimuli received during both the larval and nymphal blood meals $^{56-58}$ (FIG. 4a). The fine-tuning of c-di-GMP levels is mediated by two phosphodiesterase (Pde) proteins, PdeA and PdeB ${ }^{59,60}$ (FIG. 4a).

\section{The Borrelia-tick interface}

Larval acquisition. Lyme disease spirochaetes disseminate in their reservoir hosts and are then acquired by a naive larva taking a blood meal. Live imaging of disseminated borreliae in the dermis of mice revealed bacteria that appear to be searching randomly for the chemotactic stimuli that are provided, or elicited intracutaneously, by larval feeding ${ }^{61}$. Real-time visualization has also caught spirochaetes in the act of migrating directly towards the feeding site and rapidly entering the hypostome, whereas other spirochaetes nearby seem oblivious to the same chemoattractants (L. Bockenstedt, personal communication). Larval acquisition of spirochaetes occurs rapidly. The bacteria can be detected in larval midguts by immunofluorescence within 24 hours of attachment and are plentiful by 48 hours, before significant amounts of blood have been imbibed ${ }^{62}$.

During infection of the mammalian host, the borrelial Rrp2-RpoN-RpoS pathway is fully activated, promoting the expression of genes that are required within the mammalian host while repressing genes that are required within the tick ${ }^{37,38,41}$ (FIG. 4b). By the time the tick larvae are fully engorged, this pathway is inactive, or nearly so, resulting in the upregulation of tick-phase genes, such as $o s p A$, which are repressed by $\operatorname{RpoS}^{38,63}$. B. burgdorferi specifically binds the neuroendocrine stress hormones 


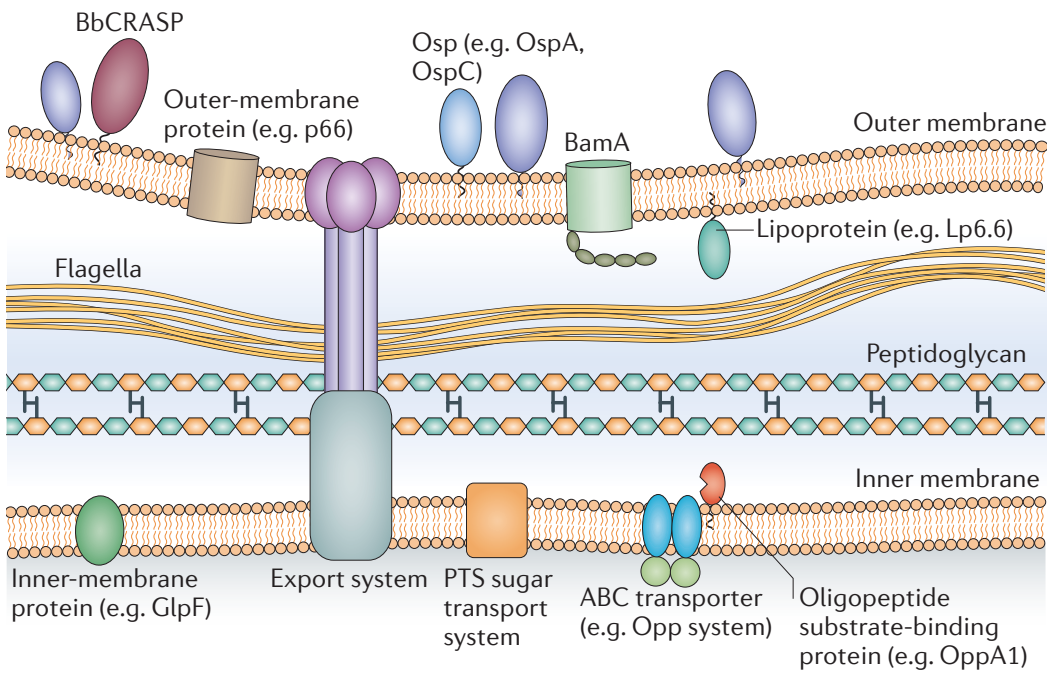

Figure 3 | The borrelial cell envelope. This schematic of the borrelial cell envelope shows the outer membrane, flagellar filaments, peptidoglycan and inner membrane. The outer membrane contains outer-surface lipoproteins (Osps) in high density and $\beta$-barrel outer-membrane-spanning proteins such as BamA in low density. The inner membrane is rich in integral membrane proteins, many of which are transporters. BbCRASP, complement regulator-acquiring surface protein; OppA1, oligopeptide permease A1; PTS, phosphotransferase system.

adrenaline and noradrenaline; it has been proposed that OspA expression is upregulated in response to the production of these hormones in the skin, which is in turn induced by the combined mechanical and pharmacological assault of larval feeding ${ }^{64}$. OspA-deficient spirochaetes cannot bind to tick receptor for OspA (TROSPA) on tick midgut epithelial cells and are eventually expelled from the larval digestive tract with the blood meal waste $^{65-67}$. In addition, activation of the Hk1-Rrp1 pathway during larval feeding (FIG. 4b), with consequent production of c-di-GMP (FIG. 4a), is required for successful bacterial colonization of the larva $e^{56-58}$. Hk1-deficient and Rrp1-deficient mutants are destroyed early during feeding ${ }^{56,57}$, perhaps because they cannot remodel their surfaces to protect themselves against antimicrobial substances elaborated by the larval midgut.

The ingress of blood triggers a burst of spirochaete replication that continues for weeks through the moult ${ }^{68}$. Following tick drop-off, the spirochaete must turn to alternative carbon sources for glycolysis and phospholipid biosynthesis, as the glucose supply within the tick midgut lumen diminishes. The expression of genes within the glp operon, which encodes proteins involved in the uptake and utilization of glycerol, is upregulated during the tick phase of the spirochaete life cycle ${ }^{57,69}$. The induction of $g l p$ expression during larval acquisition of spirochaetes is driven by activation of the Hk1-Rrp1 pathway ${ }^{57}$ and by the loss of RpoS-mediated gene repres$\operatorname{sion}^{69}$ (FIG. 4b). Ixodes spp. ticks surround the blood meal with an acellular matrix of glycoproteins and chitin, called the peritrophic membrane ${ }^{68} . N$-acetylglucosamine and chitobiose, secreted by epithelial cells for synthesis and remodelling of the peritrophic membrane, may be alternative carbon sources in addition to being essential for peptidoglycan biosynthesis ${ }^{25,70}$.
The flat nymph. Spirochaetes within flat, or unfed, nymphs exist in a poorly understood metabolic state that enables them to endure prolonged periods of nutrient deprivation ${ }^{68}$. In this state, both the Rrp2RpoN-RpoS and Hk1-Rrp1 pathways are inactive, as are mammalian-phase genes, while tick-phase genes are maximally expressed ${ }^{3738,68}$ (FIG. 4b). Despite appearances, spirochaetes residing within flat ticks are not dormant; the bacteria in dissected unfed midguts are motile, albeit sluggish ${ }^{71}$. Moreover, microarray analyses of spirochaetes cultivated in vitro under 'unfed-tick' conditions, as well as quantitative reverse transcriptase PCR (qRT-PCR) of selected genes in borreliae residing in flat ticks, suggest that many borrelial genes are preferentially expressed in the anoxic environment of the unfed midgut ${ }^{68,72-74}$. A stringent response analogous to that observed in E. coli during amino acid starvation has been suggested to occur during this phase, as the levels of transcripts encoding a RelA-SpoT orthologue are higher in bacteria cultivated in vitro under conditions that mimic unfed ticks than in bacteria cultivated under conditions that mimic fed ticks and mammals ${ }^{72}$. Because the spirochaetes in unfed ticks are not replicating, they do not require $\mathrm{N}$-acetylglucosamine. They do, however, require a carbon source to maintain subsistence levels of glycolysis. Glycerol, which is thought to be produced as a natural antifreeze by overwintering ticks ${ }^{69}$, could permeate the outer membrane of the spirochaete and enter the bacterial cytoplasm via glycerol uptake facilitator GlpF, which is expressed by borreliae in unfed ticks ${ }^{69}$. In addition to OspA, the surface-exposed lipoprotein $\mathrm{BptA}^{75}$ and the Dps-like bacterioferritin orthologue BB0690 (REF. 27) are essential for prolonged residence in flat ticks.

The nymphal blood meal. The nymphal blood meal ends a long nutritional drought but also presents the bacterium with a new and complex set of challenges. To take advantage of the influx of nutrients, the spirochaete must sense the new environment and extensively revamp its substrate uptake mechanisms and intermediary metabolism from famine to feast mode ${ }^{24,68}$. Whereas a temperature shift during cultivation in vitro induces many of the antigenic changes associated with nymphal feeding in borreliae $e^{3738,41,68}$, temperature shifting of infected unfed ticks does not ${ }^{76}$. Metabolic changes in the spirochaete that are associated with nutrition from the blood meal, and possibly components of the blood meal itself, are required to fully activate the genetic programmes associated with transmission ${ }^{48,74}$.

During the first 24 hours of nymphal feeding (the preparatory phase of the digestive process), little to no blood enters the midgut, and spirochaete numbers remain essentially unchanged from numbers in the unfed-tick state $^{71,77}$. Activation of the Rrp2-RpoN-RpoS pathway at the outset of feeding (FIG. 4b) results in the transcription of genes that are required for mammalian infection and simultaneously begins the slow repression of tickphase genes $s^{37,38,63,68}$. Nymphal feeding also induces the expression of genes which are regulated independently of RpoS and are dependent instead on $\sigma^{70}$ (also known as RpoD). The products encoded by these genes include 
a

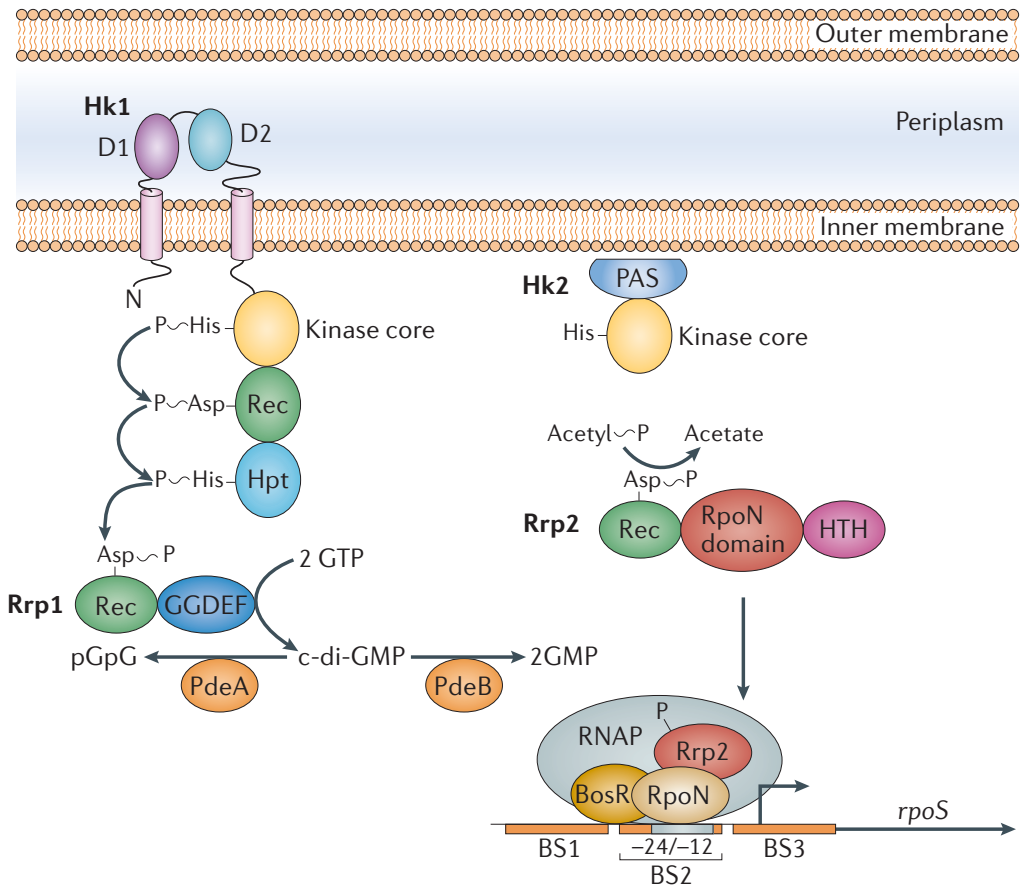

b

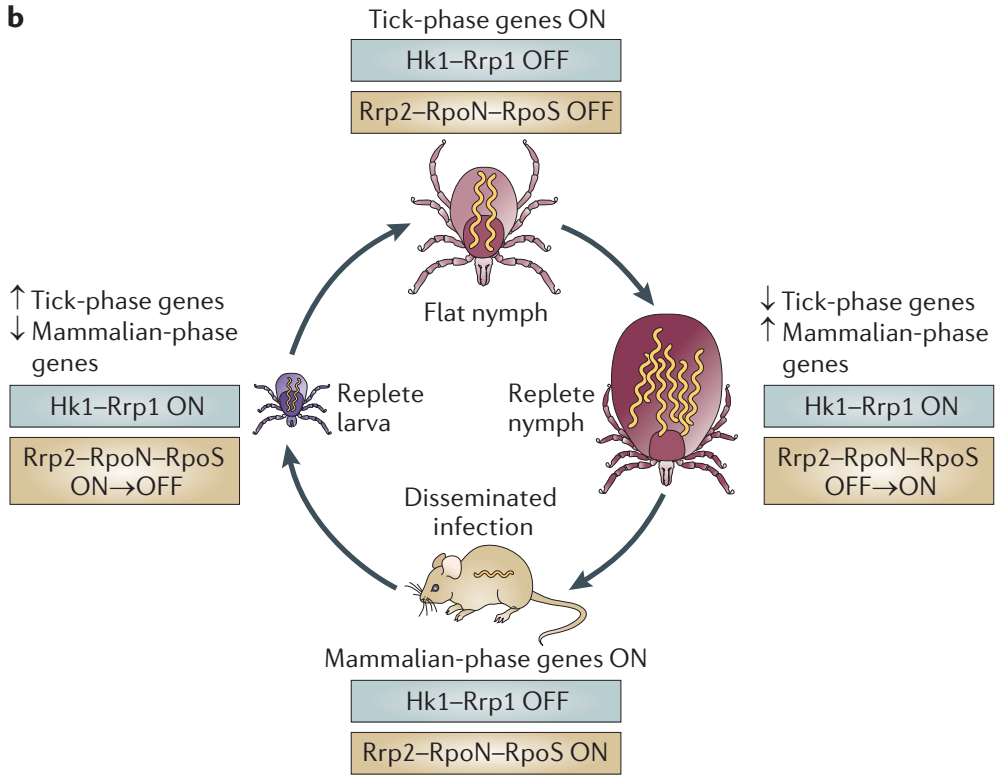

Complement factor $\mathrm{H}$ and complement factor $\mathrm{H}$-like protein 1

Serum proteins that prevent inadvertent activation of the alternative complement pathway. Binding of these proteins by BbCRASPs (complement regulatoracquiring surface proteins) on the surface of Lyme disease spirochaetes protects the bacteria against the lytic activity of complement generated via the alternative pathway.

the outer-membrane-spanning protein p66, which acts as a porin and adhesin ${ }^{78-80}$, and BbCRASPs (complement regulator-acquiring surface proteins), which are outersurface lipoproteins that protect the bacterium against complement-mediated lysis by binding complement factor $\mathrm{H}$ and complement factor $\mathrm{H}$-like protein 1 (REFS 81-85). As is the case during larval feeding, spirochaetes require the two-component system Hk1-Rrp1 to avoid being destroyed at the start of nymphal feeding ${ }^{56,57}$ (FIG. 4b).

By 48 hours, bacterial replication and dissemination are well under way ${ }^{68,71,77,86,87}$. Although it has been suggested that spirochaetes traverse the tick midgut by downregulating OspA, detaching from epithelial cells, and then using their motility to migrate between cells and penetrate the basement membrane, two lines of
Figure 4 | Regulation of gene expression in Borrelia burgdorferi. a | The histidine kinase 1 (Hk1)-response regulatory protein 1 (Rrp1) and the alternative RNA polymerase $\sigma$-factor RpoS global regulatory systems. Binding of unidentified ligands to the periplasmic sensor domains (D1 and D2) of the hybrid histidine kinase Hk1 initiates a phosphorelay that activates the diguanylyl cyclase activity of Rrp1, resulting in the production of cyclic di-GMP (c-di-GMP) ${ }^{56-58,131}$. Phosphodiesterase A (PdeA) and PdeB degrade c-di-GMP to 5'-phosphoguanylyl$\left(3^{\prime}-5^{\prime}\right)$-guanosine (pGpG) and GMP, respectively ${ }^{59,60}$. Activation of Rrp2 in vitro and in vivo occurs via the high-energy phosphoryl donor acetyl-phosphate rather than by its presumptive cognate histidine kinase, Hk2 (REF. 48). The function of Hk2 is currently unknown. Phosphorylated Rrp2, Borrelia oxidative stress regulator (BosR) and RpoN initiate transcription of $\mathrm{rpoS}^{37,38,42-47}$. This is depicted as a trimeric complex, but the precise interactions between these proteins have yet to be determined. Putative BosR-binding sites (BSs) containing the direct repeat sequence TAAATTAAAT are shown ${ }^{47}$; $-24 /-12$ is the RpoN-binding site in the rpoS promoter ${ }^{47}$. RpoS in turn induces the expression of genes that are required during the mammalian-host phase of the spirochaete life cycle and represses the expression of tick-phase genes. $\mathbf{b}$ | Expression of the Hk1-Rrp1 and RpoS global regulatory systems during the $B$. burgdorferi life cycle $^{37,38,56-58,68,80}$. In the flat nymph, both the Hk1-Rrp1 and the Rrp2-RpoN-RpoS systems are inactive and only tick-phase genes are expressed. The nymphal blood meal activates both the Hk1-Rrp1 and Rrp2-RpoN-RpoS pathways. Expression of mammalian-phase genes begins in concert with downregulation of tick-phase genes. Following inoculation into a mammalian host, the spirochaetes complete the process of adaptation; the Hk1-Rrp1 pathway is inactive, the Rrp2-RpoN-RpoS pathway is active, mammalian-phase genes are expressed and tick-phase genes are repressed. During larval acquisition of spirochaetes, Hk1-Rrp1 is activated, probably at the feeding site, whereas the Rrp2-RpoNRpoS system is inactivated. Mammalian-phase genes are repressed, expression of tick-phase genes begins and ingested spirochaetes bind to the larval midgut epithelium via OspA and possibly other receptors ${ }^{65-67}$. GGDEF, a conserved motif present in diguanylyl cyclases ${ }^{131}$; $\mathrm{Hpt}$, histidine-containing phosphotransfer domain ${ }^{56}$; $\mathrm{HTH}$, helix-turn-helix domain; N, amino; PAS, putative sensor domain for Hk2; Rec, receiver domain. evidence argue that this scenario is overly simplistic. First, OspA is highly expressed by spirochaetes distributed throughout the midgut, even at late time points during feeding ${ }^{62,63,87}$. Second, confocal microscopy has shown that the bacteria maintain a close association with the epithelial cells as they replicate, coalescing into networks of non-motile bacteria that literally surround the hypertrophying epithelial cells and progress towards the basement membrane $e^{71,86}$. Spirochaetes that reach the basolateral surface of the epithelium transition into motile organisms that cross the basement membrane and enter the haemocoel ${ }^{71,88}$. BBE31, a surface-exposed borrelial lipoprotein, may be required for spirochaetes to penetrate the midgut through its interaction with TRE31, a tick protein that is secreted by epithelial cells 


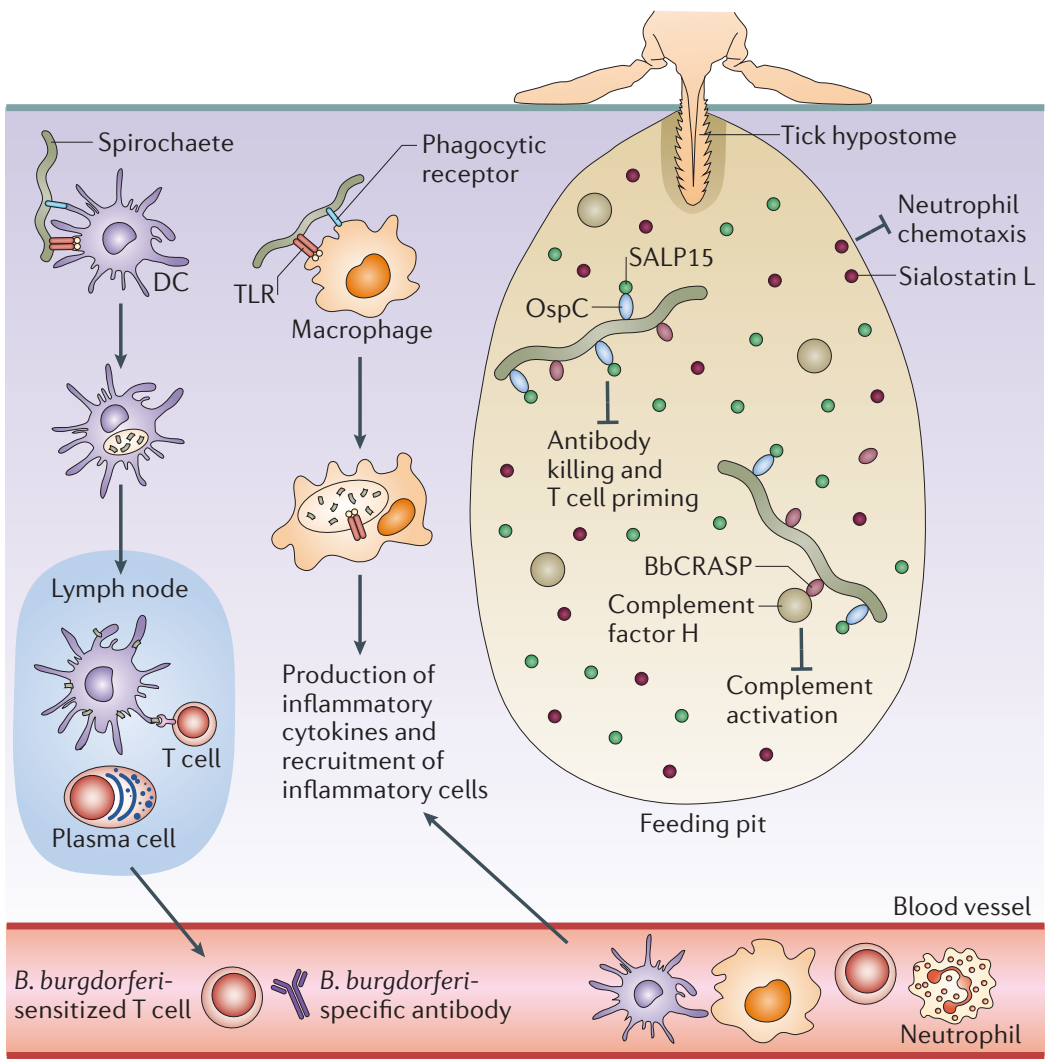

Figure 5 | The tick-mammal interface. The tick creates a feeding pit with its mouthparts, using its hypostome (a barbed protuberance) as an anchor to the skin of its host. Initial salivary secretions form a cement cone around the hypostome that further anchors the tick during feeding. Subsequently, the tick produces copious amounts of saliva containing a plethora of bioactive agents that Borrelia burgdorferi sensu lato exploits to help establish infection. The diagram shows just a few of these bioactive agents, including SALP15 (which binds to the spirochaetes and inhibits killing of the bacteria by antibodies, as well as T cell priming within the lymph nodes) and sialostatin L (which blocks neutrophil chemotaxis) ${ }^{68,92,93}$. A group of borrelial surface lipoproteins, collectively referred to as BbCRASPs (complement regulator-acquiring surface proteins), bind complement factor $\mathrm{H}$, preventing activation of the alternative complement pathway ${ }^{81-84}$. B. burgdorferi cells are recognized by innate immune effector cells such as dendritic cells (DCs), neutrophils and macrophages, initially via surface-exposed pattern recognition receptors; activation of these cells increases following internalization and degradation of spirochetes within phagolysosomes. DCs that have taken up spirochaetes migrate to the lymph nodes, where they present processed borrelial antigens to T cells and B cells. Sensitized T cells enter the circulation and are recruited to the site of infection. Plasma cells secrete specific antibodies that can kill B. burgdorferi via complementdependent and -independent pathways. Production of pro-inflammatory cytokines by activated macrophages results in the recruitment of additional neutrophils, T cells, macrophages and DCs to the bite site, and eventually the development of erythema migrans $^{15}$. OspC, outer-surface lipoprotein C; TLR, Toll-like receptor.

Basement membrane A collagenous matrix that encloses the surface of the tick midgut facing the haemocoel.

\section{Haemocoel}

The fluid-filled space that surrounds the tick midgut and contains the salivary glands. during feeding ${ }^{89}$. Binding of host-derived molecules, such as plasminogen, to the bacterial surface probably facilitates penetration of the collagenous matrix of the basement membrane ${ }^{90}$. The salivary glands pose the final mechanical barrier to be overcome; only a handful of spirochaetes reach the interior of the gland, where they access the salivary stream ${ }^{71,77,87}$.

\section{Within the mammalian host}

The bite site. The host factors in tick saliva that enhance the survival of B. burgdorferi and are inoculated into the cutaneous bite site (FIG. 5) include molecules that can impede various mammalian responses, such as the generation of reactive oxygen species (ISL 1373), activation of complement (ISAC and SALP20), chemotaxis of neutrophils (sialostatin L), and antibody-mediated killing, dendritic cell-mediated priming of T cells and keratinocyte-mediated release of cytokines and antimicrobial peptides (SALP15) ${ }^{68,91-93}$. Expression of the bacterial protein $\mathrm{OspC}$ is crucial for the establishment of early infection ${ }^{94-96}$, although there is controversy as to whether OspC promotes the penetration of salivary glands by spirochaetes disseminating within the tick or the survival of spirochaetes deposited at the bite site through binding of SALP15. As in the feeding nymph, borrelial proteins that bind to complement factor $\mathrm{H}$ - and complement factor $\mathrm{H}$-like protein 1 protect the bacterium against complement-mediated killing during this early window of vulnerability ${ }^{80-84}$.

Initial sensing of B. burgdorferi by the mammalian host probably occurs through pattern recognition receptors, such as Toll-like receptors (TLRs) and NOD-like receptors (NLRs), on dendritic cells and sentinel macrophages within the dermis (FIG. 5). TLR2, which heterodimerizes with TLR1 to recognize triacylated lipoproteins, seems to be central to the induction of many inflammatory cytokines in response to B. burgdorferi. Engagement of TLR2-TLR1 heterodimers by borrelial lipoproteins results in activation of mitogen-activated protein kinase (MAPK) pathways, translocation of nuclear factor- $\kappa \mathrm{B}$ (NF- $\kappa \mathrm{B})$ into the nucleus, and subsequent production and release of inflammatory mediators ${ }^{15}$. Other TLRs, such as TLR5, TLR7, TLR8 and TLR9, cooperate with TLR2-TLR1 to induce pro-inflammatory molecules, including type I interferons (IFNs) ${ }^{97-100}$. TLR-mediated release of chemokines and cytokines by resident phagocytes presumably attracts other inflammatory cells to the tick bite site. In mice, neutrophils are recruited early but disappear within 16 hours of inoculation and are followed by monocytes and macrophages, both of which are capable killers of $B$. burgdorfer $i^{15}$. However, B. burgdorferi is a highly motile organism and can move at speeds of up to $4 \mu \mathrm{m} \mathrm{sec}^{-1}$ in tissue ${ }^{61}$, enabling it to evade capture by these comparatively sluggish professional phagocytes. Spirochaetes that are unable to evade phagocytes are ingested and rapidly degraded within phagosomal vacuoles ${ }^{98}$. The phagocytosis of borreliae and subsequent degradation within phagolysosomes further amplifies the release of inflammatory cytokines through activation of TLR signalling within phagolysosomes ${ }^{98}$. Internalization of spirochaetes in the absence of antibodies is mediated by at least two different integrins, $\alpha \mathrm{M} \beta 2$ integrin (also known as CR3 or CD18-CD11b) and $\alpha 3 \beta 1$ integrin ${ }^{15,101}$. Although the internalization of B. burgdorferi results in activation of the inflammasome, neither the course of disease nor the spirochaete burden is worsened appreciably in caspase 1-deficient mice ${ }^{15}$. In humans, recruitment of T cells heralds the development of the characteristic erythema migrans rash (FIC 1). Biopsies of erythema migrans lesions show infiltration by T cells (CD8 ${ }^{+}$cells as well as $\mathrm{CD}^{+}$cells), macrophages, plasmacytoid and monocytoid dendritic cells, and neutrophils 
in varying proportions, but no B cells ${ }^{102,103}$; a number of inflammatory cytokines and chemokines have also been identified in erythema migrans lesions ${ }^{102,104}$.

Dissemination and immune evasion. After a delay of up to 2 days, B. burgdorferi begins to spread to distant tissues ${ }^{105}$. To disseminate, B. burgdorferi penetrates the matrix between cells and enters capillary beds. The spirochaete circumvents its inability to produce enzymes that are capable of digesting extracellular matrix components by appropriating host proteases such as plasminogen and its activator, urokinase ${ }^{88,106}$. B. burgdorferi also induces multiple host matrix metalloproteinases (MMPs), the major class of host proteases involved in the degradation of extracellular matrix components, from both phagocytic and non-phagocytic cells ${ }^{15,80}$. Entry into capillaries provides $B$. burgdorferi with access to the bloodstream. Egress from the circulation into tissues involves tethering and adhesion to vascular endothelium followed by extravasation ${ }^{61}$. B. burgdorferi expresses a variety of adhesins that could mediate attachment to host tissues through diverse receptors such as integrins (which can bind p66 and the outer-surface protein encoded by the locus BB_B07 ${ }^{78,107}$, proteoglycans (which can bind decorin-binding protein $\mathrm{A}(\mathrm{DbpA})$ and $\mathrm{DbpB})^{108}$, glycosaminoglycans ${ }^{109}$, laminin (which can bind ErpX and $\mathrm{BmpA})^{110,111}$ and fibronectin (which can bind the protein encoded by the locus BB_K32 and RevA) $)^{80,112,113}$.

Carditis in mice and humans is predominantly caused by infiltration of monocytes or macrophages, with a minority of lymphocytes and neutrophils ${ }^{15,17}$. Arthritis in susceptible strains of mice, by comparison, shows a predominance of neutrophils with smaller numbers of macrophages and lymphocytes ${ }^{15}$. IFN $\gamma$ appears to mediate carditis, but not arthritis, in susceptible mice ${ }^{114,115}$, whereas type I IFNs may be particularly important for the development of arthritis ${ }^{99}$. B. burgdorferi is unique among pathogens in that its diacylglycerol glycolipid can directly activate invariant natural killer $T$ cells ${ }^{116}$. These $\mathrm{T}$ cells might participate in clearing spirochaetaemia after being recruited by Kupffer cells that recognize $B$. burgdorferi in the liver ${ }^{117}$. In mice, invariant natural killer T cells are recruited to the heart during B. burgdorferi infection, where they could have a role in controlling infection and inflammation through augmenting phagocytosis $^{114}$.

Both the innate and adaptive immune systems are important for controlling infection and inflammation during the disseminated phase, as mice that are deficient in either system have greater bacterial burdens than wild-type mice ${ }^{15}$. T cells do not appear to be required for resolution of either arthritis or carditis in mice; in fact, the presence of $\mathrm{T}$ cells without $\mathrm{B}$ cells can worsen both ${ }^{118}$. However, $\mathrm{CD} 4^{+} \mathrm{T}$ cells can hasten resolution of carditis in the presence of $\mathrm{B}$ cells ${ }^{118}$. The development of the specific humoral response to B. burgdorferi is crucial for clearing the pathogen ${ }^{15,119}$. Early T cell-independent production of antibodies, predominantly immunoglobulin $\mathrm{M}$, is crucial for the initial reduction of spirochaete burdens ${ }^{120,121}$. T cell-dependent production of immunoglobulin $\mathrm{G}$ by B cells is typically detectable by the second week of infection ${ }^{15}$. Antibody production does not seem to require TLR signalling, as both TLR2-deficient and MYD88-deficient mice develop normal humoral responses to infection with B. burgdorferi ${ }^{121-123}$. Antibodies against many different borrelial proteins elicit strain-dependent reductions in pathogen burden or a reduction in carditis or arthritis ${ }^{15}$. In response, the bacterium is thought to downregulate lipoproteins, such as OspC, which are no longer required to establish or maintain infection ${ }^{96,124}$. B. burgdorferi also uses a system of antigenic variation to evade antibodies. VlsE is a $35 \mathrm{kDa}$ lipoprotein that undergoes antigenic variation through the recombination of sequences from silent cassettes into the expressed $v l s E$ locus ${ }^{84,125,126}$. The spirochaete may also exploit mechanisms that are used by the host to suppress inflammation and therefore limit tissue damage, in effect making the mammalian host an unwitting accomplice to the spirochaete's strategy for persistence $^{127}$.

\section{Concluding remarks and future directions}

Two facets of the life cycle of B. burgdorferi - the bacterium's ability to adapt to markedly divergent host environments and its ability to evade the defences of its mammalian reservoir - account, to a large extent, for the extraordinary zoonotic success of this spirochaete and its continued expansion as a threat to public health. We are only just beginning to understand the mechanisms whereby this versatile bacterium coordinates changes in its transcriptome, cellular architecture and metabolism with the feeding behaviour and physiology of its arthropod vector. Marginally better understood are the stratagems that enable the spirochaete to navigate within the mammal, regularly gaining entry to niches that are impassable for other bacteria, when establishing a persistent infection. Despite its small genome, the Lyme disease spirochaete possesses deceptively complex machinery for tightly regulating gene and protein expression, with an unusual combination of components identified to date. The small number of orthologues for known transcription factors hints at the existence of novel post-transcriptional control pathways; it is noteworthy that the burgeoning interest in small RNAs among microbiologists has only recently made its influence felt in the field of borrelial research. Co-evolution of the spirochaete with its arthropod and mammalian hosts has enabled the bacterium to take advantage of host physiological processes and make compensatory reductions in its own biosynthetic machinery. In so doing, the bacterium has had to develop ingenious and parsimonious strategies to obtain and utilize the nutrient sources available within the 'feast and famine' confines of its life cycle. Indeed, the nutrients themselves, especially carbon sources, and/or their metabolic by-products seem to provide regulatory as well as chemotactic signals that guide the spirochaete as it moves between hosts.

Beginning with the discovery that spirochaete lipoproteins are major inflammatory agonists, we have learned a great deal about the mechanisms by which the mammalian host senses the presence of live spirochaetes and mobilizes cellular and humoral defences to combat 
the intruder. By contrast, little is understood about the processes that occur late in infection and the mechanisms that enable the bacterium to persist in the face of the robust cellular and humoral immune responses that it elicits. In the past, the emphasis has been on immune evasion by the spirochaete, and this topic needs far more scrutiny. Nevertheless, attention also needs to be paid to the possibility that host-mediated modulation of pathogen-sensing pathways and of resultant responses contributes to spirochaete persistence. We are just beginning to understand the polymorphisms in specific mouse genes that result in some inbred strains of mice being more prone to manifestations of infection than the natural P. leucopus host. Whether these genes are identical to those responsible for disease susceptibility and expression in humans is unknown. However, one can envision translational studies to answer the question of why some infected patients have only subclinical disease, whereas others develop overt manifestations. These and other advances will hopefully lead to a better understanding of the determinants of vector and host specificity for B. burgdorferi, and to the manipulation of these determinants to interrupt the cycle of transmission. Although eliminating Lyme disease spirochaetes from nature is unrealistic, diminishing their threat to humans would seem to be an achievable goal.
1. Benach, J. L. \& Garcia Monco, J. C. in Borrelia: Molecular Biology, Host Interaction, and Pathogenesis (eds Samuels, D. S. \& Radolf, J. D.) 7-26 (Caister Academic, Norfolk, UK, 2010).

2. Steere, A. C. et al. Lyme arthritis: an epidemic of oligoarticular arthritis in children and adults in three Connecticut communities. Arthritis Rheum. 20, 7-17 (1977).

An article describing the original epidemiologica study of the outbreak of arthritis in and around Lyme, Connecticut.

3. Burgdorfer, W. et al. Lyme disease-a tick-borne spirochetosis? Science 216, 1317-1319 (1982) A classic paper that provides the first convincing evidence that Lyme disease is a tick-borne illness. Steere, A. C. et al. The spirochetal etiology of Lyme disease. N. Engl. J. Med. 308, 733-740 (1983).

5. Benach, J. L. et al. Spirochetes isolated from the blood of two patients with Lyme disease. N. Engl. J. Med. 308, 740-742 (1983).

This and reference 4 are the first two reports to describe the isolation of Lyme disease spirochaetes from patients.

6. Hyde, F. W. \& Johnson, R. C. Genetic relationship of Lyme disease spirochetes to Borrelia, Treponema, and Leptospira spp. J. Clin Microbiol. 20, 151-154 (1984).

7. Piesman, J. \& Schwan, T. G. in Borrelia: Molecular Biology, Host Interaction, and Pathogenesis (eds Samuels, D. S. \& Radolf, J. D.) 251-278 (Caister Academic, Norfolk, UK, 2010).

8. Hall-Baker, P. A. et al. Summary of notifiable diseases - United States, 2008. Morb. Mortal. Wkly Rep. 57 1-94 (2010).

9. O'Connell, S., Granstrom, M., Gray, J. S. \& Stanek, G. Epidemiology of European Lyme borreliosis. Zentralbl. Bakteriol. 287, 229-240 (1998).

10. Kurtenbach, K. et al. Fundamental processes in the evolutionary ecology of Lyme borreliosis. Nature Rev. Microbiol. 4, 660-669 (2006).

11. Mather, T. N. \& Mather, M. E. Intrinsic competence of three ixodid ticks (Acari) as vectors of the Lyme disease spirochete. J. Med. Entomol. 27, 646-650 (1990).

12. Ribeiro, J. M., Mather, T. N., Piesman, J. \& Spielman, A Dissemination and salivary delivery of Lyme disease spirochetes in vector ticks (Acari: Ixodidae). J. Med. Entomol. 24, 201-205 (1987).

A definitive demonstration that spirochaetes are transmitted by the salivary route during tick feeding.

13. Piesman, J., Mather, T. N., Sinsky, R. J. \& Spielman, A Duration of tick attachment and Borrelia burgdorferi transmission. J. Clin. Microbiol. 25, 557-558 (1987) A classic study that defines the $\sim 48$ hour window required for transmission of spirochaetes by feeding nymphs.

14. Barthold, S. W., Cadavid, D. \& Philipp, M. T. in Borrelia: Molecular Biology, Host Interaction, and Pathogenesis (eds Samuels, D. S. \& Radolf, J. D.) 359-411 (Caister Academic, Norfolk, UK, 2010).

15. Weis, J. J. \& Bockenstedt, L. K. in Borrelia: Molecular Biology, Host Interaction, and Pathogenesis (ed. Samuels, D. S. \& Radolf, J. D.) 413-441 (Caister Academic, Norfolk, UK, 2010).

16. Fraser, C. M. et al. Genomic sequence of a Lyme disease spirochaete, Borrelia burgdorferi. Nature 390, 580-586 (1997).
A sequence analysis of the $B$. burgdorferi chromosome; one of the first genomic-sequence papers for this species.

17. Radolf, J. D., Salazar, J. C. \& Dattwyler, R. J. in Borrelia: Molecular Biology, Host Interaction, and Pathogenesis (eds Samuels, D. S. \& Radolf, J. D.) 487-533 (Caister Academic, Norfolk, UK, 2010).

18. Fahrer, H. et al. The prevalence and incidence of clinical and asymptomatic Lyme borreliosis in a population at risk. J. Infect. Dis. 163, 305-310 (1991).

19. Wormser, G. P. et al. Brief communication: hematogenous dissemination in early Lyme disease. Ann. Intern. Med. 142, 751-755 (2005)

20. Stanek, G. \& Strle, F. Lyme disease: European perspective. Infect. Dis. Clin. North Am. 22, 327-339, (2008).

21. Wormser, G. P. et al. Borrelia burgdorferi genotype predicts the capacity for hematogenous dissemination during early Lyme disease. J. Infect. Dis. 198 1358-1364 (2008)

22. Earnhart, C. G., Buckles, E. L., Dumler, J. S. \& Marconi, R. T. Demonstration of OspC type diversity in invasive human Lyme disease isolates and identification of previously uncharacterized epitopes that define the specificity of the OspC murine antibody response. Infect. Immun. 73, 7869-7877 (2005).

23. Casjens, S. R., Eggers, C. H. \& Schwartz, I. in Borrelia: Molecular Biology, Host Interaction, and Pathogenesis (eds Samuels, D. S. \& Radolf, J. D.) 27-53 (Caister Academic, Norfolk, UK, 2010).

24. Gheradini, F., Boylan, J., Lawrence, K. \& Skare, J. in Borrelia: Molecular Biology, Host Interaction, and Pathogenesis (eds Samuels, D. S. \& Radolf, J. D.) 103-138 (Caister Academic, Norfolk, UK, 2010).

25. von Lackum, K. \& Stevenson, B. Carbohydrate utilization by the Lyme borreliosis spirochete, Borrelia burgdorferi. FEMS Microbiol. Lett. 243, 173-179 (2005).

26. Posey, J. E. \& Gherardini, F. C. Lack of a role for iron in the Lyme disease pathogen. Science 288, 1651-1653 (2000).

An article that provides evidence for one of the most unusual physiological properties of $B$. burgdorferi: its lack of a requirement for iron.

27. Li, X. et al. The Lyme disease agent Borrelia burgdorferi requires BB0690, a Dps homologue, to persist within ticks. Mol. Microbiol. 63, 694-710 (2007).

28. Bergstrom, S. \& Zuckert, W. R. in Borrelia: Molecular Biology, Host Interaction, and Pathogenesis (eds Samuels, D. S. \& Radolf, J. D.) 139-166 (Caister Academic, Norfolk, UK, 2010)

29. LaRocca, T. J. et al. Cholesterol lipids of Borrelia burgdorferi form lipid rafts and are required for the bactericidal activity of a complement-independent antibody. Cell Host Microbe 8, 331-342 (2010). A technically innovative study relating antibody killing of spirochaetes to the unique lipid composition and physical properties of the spirochaete outer membrane.

30. Schroder, N. W. et al. Acylated cholesteryl galactoside as a novel immunogenic motif in Borrelia burgdorferi sensu stricto. J. Biol. Chem. 278, 33645-33653 (2003).

31. Bunikis, I. et al. An RND-type efflux system in Borrelia burgdorferi is involved in virulence and resistance to antimicrobial compounds. PLoS Pathog. 4, e1000009 (2008).

32. Lenhart, T. R. \& Akins, D. R. Borrelia burgdorferi locus BB0795 encodes a BamA orthologue required for growth and efficient localization of outer membrane proteins. Mol. Microbiol. 75, 692-709 (2010).

33. Goldstein, S. F. et al. in Borrelia: Molecular Biology, Host Interaction, and Pathogenesis (eds Samuels, D. S. \& Radolf, J. D.) 167-187 (Caister Academic, Norfolk, UK, 2010)

34. Liu, J. et al. Intact flagellar motor of Borrelia burgdorferi revealed by cryo-electron tomography: evidence for stator ring curvature and rotor/C-ring assembly flexion. J. Bacteriol. 191, 5026-5036 (2009).

35. Charon, N. W. et al. The flat-ribbon configuration of the periplasmic flagella of Borrelia burgdorferi and its relationship to motility and morphology. J. Bacteriol. 191, 600-607 (2009).

36. Xu, H., Raddi, G., Liu, J., Charon, N. W. \& Li, C. Chemoreceptors and flagellar motors are subterminally located in close proximity at the two cell poles in spirochetes. J. Bacteriol. 193, 2652-2656 (2011)

37. Skare, J. T., Carroll, J. A., Yang, X. F., Samuels, D. S. ¿ Akins, D. R. in Borrelia: Molecular Biology, Host Interaction, and Pathogenesis (eds Samuels, D. S. \& Radolf, J. D.) 67-101 (Caister Academic, Norfolk, UK, 2010)

38. Samuels, D. S. Gene regulation in Borrelia burgdorferi. Annu. Rev. Microbiol. 65, 479-499 (2011).

39. Hubner, A. et al. Expression of Borrelia burgdorfer OspC and DbpA is controlled by a RpoN-RpoS regulatory pathway. Proc. Natl Acad. Sci. USA 98 12724-12729 (2001).

A ground-breaking demonstration of the unique control mechanism for rpos transcription in $B$. burgdorferi and the role of this alternative $\sigma$-factor in controlling virulence gene expression.

40. Fisher, M. A. et al. Borrelia burgdorferi $\sigma^{54}$ is required for mammalian infection and vector transmission but not for tick colonization. Proc. Natl Acad. Sci. USA 102, 5162-5167 (2005)

41. Caimano, M. J. et al. Analysis of the RpoS regulon in Borrelia burgdorferi in response to mammalian host signals provides insight into RpoS function during the enzootic cycle. Mol. Microbiol. 65, 1193-1217 (2007).

42. Yang, X. F., Alani, S. M. \& Norgard, M. V. The response regulator Rrp2 is essential for the expression of major membrane lipoproteins in Borrelia burgdorferi. Proc. Natl Acad. Sci. USA 100, 11001-11006 (2003). An elegant use of mutagenesis to demonstrate the essentiality of Rrp2 for the induction of RpoS-dependent gene regulation.

43. Blevins, J. S. et al. Rrp2, a $\sigma^{54}$-dependent transcriptional activator of Borrelia burgdorferi, activates $r p o S$ in an enhancer-independent manner J. Bacteriol. 191, 2902-2905 (2009).

44. Boardman, B. K. et al. Essential role of the response regulator Rrp2 in the infectious cycle of Borrelia burgdorferi. Infect. Immun. 76, 3844-3853 (2008).

45. Burtnick, M. N. et al. Insights into the complex regulation of rpoS in Borrelia burgdorferi. $\mathrm{Mol}$. Microbiol. 65, 277-293 (2007). 
46. Hyde, J. A., Shaw, D. K., Smith, R. III, Trzeciakowski, J. P. \& Skare, J. T. The BosR regulatory protein of Borrelia burgdorferi interfaces with the RpoS regulatory pathway and modulates both the oxidative stress response and pathogenic properties of the Lyme disease spirochete. Mol. Microbiol. 74 1344-1355 (2009).

One of the first investigations to demonstrate that the borrelial Fur-PerR orthologue, BosR, is required for expression of $r p o S$.

47. Ouyang, Z., Deka, R. K. \& Norgard, M. V. BosR (BB0647) controls the RpoN-RpoS regulatory pathway and virulence expression in Borrelia burgdorferi by a novel DNA-binding mechanism. PLoS Pathog. 7 , e 1001272 (2011)

An impressive work that defines the binding-site motif for BosR near the rpoS promoter

48. Xu, H. et al. Role of acetyl-phosphate in activation of the Rrp2-RpoN-RpoS pathway in Borrelia burgdorfer PLoS Pathog. 6, e1001104 (2010).

A study that elegantly relates the concept of the acetate switch in $E$. coli to the activation of Rrp2 and differential gene expression in B. burgdorferi.

49. Lybecker, M. C. \& Samuels, D. S. Temperature-induced regulation of RpoS by a small RNA in Borrelia burgdorferi. Mol. Microbiol. 64, 1075-1089 (2007). The first paper to present evidence for post-transcriptional regulation of $r p o S$ in B. burgdorferi.

50. Lybecker, M. C., Abel, C. A., Feig, A. L. \& Samuels, D. S. Identification and function of the RNA chaperone $\mathrm{Hfq}$ in the Lyme disease spirochete Borrelia burgdorferi. Mol. Microbiol. 78, 622-635 (2010).

51. Karna, S. L. et al. CsrA modulates levels of lipoproteins and key regulators of gene expression critical for pathogenic mechanisms of Borrelia burgdorferi. Infect. Immun. 79, 732-744 (2011).

52. Sze, C. W. \& Li, C. Inactivation of bb0184, which encodes carbon storage regulator $\mathrm{A}$, represses the infectivity of Borrelia burgdorferi. Infect. Immun. 79, 1270-1279 (2011)

53. Jutras, B. L. et al. BpaB and EbfC DNA-binding proteins regulate production of the Lyme disease spirochete's infection-associated Erp surface proteins. J. Bacteriol. 9 Dec 2011 (doi: 10.1128/JB.06394-11)

54. Medrano, M. S. et al. Regulators of expression of the oligopeptide permease A proteins of Borrelia burgdorferi. J. Bacteriol. 189, 2653-2659 (2007)

55. Medrano, M. S., Policastro, P. F., Schwan, T. G. \& Coburn, J. Interaction of Borrelia burgdorferi $\mathrm{Hbb}$ with the $p 66$ promoter. Nucleic Acids Res. 38 414-427 (2010)

56. Caimano, M. J. et al. The hybrid histidine kinase Hk 1 is part of a two-component system that is essential for survival of Borrelia burgdorferi in feeding Ixodes scapularis ticks. Infect. Immun 79, 3117-3130 (2011)

The demonstration that the sensor kinase $\mathrm{Hk} 1$ is required for adaptation of the spirochaete to the tick host.

57. He, M. et al. Cyclic di-GMP is essential for the survival of Borrelia burgdorferi in ticks. PLOS Pathog. 7 , e1002133 (2011).

The discovery that the response regulator $\operatorname{Rrp} 1$ is required for adaptation of the spirochaete to the tick host.

58. Kostick, J. L. et al. The diguanylate cyclase, Rrp1, regulates critical steps in the enzootic cycle of the Lyme disease spirochetes. Mol. Microbiol. 81, 219-231 (2011).

The finding that Rrp 1 is required for adaptation of the spirochaete to the tick host.

59. Sultan, S. Z. et al. Analysis of the HD-GYP domain cyclic-di-GMP phosphodiesterase reveals a role in motility and enzootic life cycle of Borrelia burgdorferi. Infect. Immun. 79, 3273-3283 (2011).

60. Sultan, S. Z., Pitzer, J. E., Miller, M. R. \& Motaleb, M. A. Analysis of a Borrelia burgdorferi phosphodiesterase demonstrates a role for cyclicdi-guanosine monophosphate in motility and virulence. Mol Microbiol 77, 128-142 (2010).

61. Moriarty, T. J. et al. Real-time high resolution 3D imaging of the Lyme disease spirochete adhering to and escaping from the vasculature of a living host. PLoS Pathog. 4, e1000090 (2008)

A landmark paper describing in vivo imaging of $B$. burgdorferi in mice, including remarkable images and movies of spirochaetes penetrating the vascular endothelium.

62. Schwan, T. G. \& Piesman, J. Temporal changes in outer surface proteins $A$ and $C$ of the Lyme disease associated spirochete, Borrelia burgdorferi, during the chain of infection in ticks and mice. J. Clin. Microbiol. 38, 382-388 (2000).

63. Mulay, V. B. et al. Borrelia burgdorferi bba74 is expressed exclusively during tick feeding and is regulated by both arthropod- and mammalian hostspecific signals. J. Bacteriol 191, 2783-2794 (2009).

64. Scheckelhoff, M. R., Telford, S. R., Wesley, M. \& Hu, L. T. Borrelia burgdorferi intercepts host hormonal signals to regulate expression of outer surface protein A Proc. Natl Acad. Sci. USA 104, 7247-7252 (2007).

65. Pal, U et al. TROSPA, an Ixodes scapularis receptor for Borrelia burgdorferi. Cell 119, 457-468 (2004). The identification of the receptor, expressed on tick midgut epithelial cells, that is crucial for the tick phase of the spirochaete's enzootic life cycle.

66. Yang, X. F., Pal, U., Alani, S. M., Fikrig, E. \& Norgard, M. V. Essential role for OspA/B in the life cycle of the Lyme disease spirochete. J. Exp. Med. 199, 641-648 (2004).

An elegant genetic demonstration that spirochaetes lacking OspA are virulent but unable to colonize larval midguts.

67. Battisti, J. M. et al. Outer surface protein A protects Lyme disease spirochetes from acquired host immunity in the tick vector. Infect. Immun. 76 5228-5237 (2008)

68. Pal, U. \& Fikrig, E. in Borrelia: Molecular Biology, Host Interactions, and Pathogenesis (eds Samuels, D. S. \& Radolf, J. D.) 279-298 (Caister Academic Norfold, UK, 2010).

69. Pappas, C. J. et al. Borrelia burgdorferi requires glycerol for maximum fitness during the tick phase of the enzootic cycle. PLoS Pathog. 7, e1002102 (2011).

70. Tilly, K. et al. Genetics and regulation of chitobiose utilization in Borrelia burgdorferi. J. Bacteriol. 183 5544-5553 (2001).

71. Dunham-Ems, S. M. et al. Live imaging reveals a biphasic mode of dissemination of Borrelia burgdorfer within ticks. J. Clin. Invest. 119, 3652-3665 (2009). The first paper to use live imaging to track spirochaetes disseminating in feeding nymphs.

72. Revel, A. T., Talaat, A. M. \& Norgard, M. V. DNA microarray analysis of differential gene expression in Borrelia burgdorferi, the Lyme disease spirochete. Proc. Natl Acad. Sci. USA 99, 1562-1567 (2002). The first microarray analysis of the $B$. burgdorferi transcriptome, which cleverly uses in vitro conditions to emulate the tick and mammalian phases of the enzootic cycle.

73. Ojaimi, C. et al. Profiling of temperature-induced changes in Borrelia burgdorferi gene expression by using whole genome arrays. Infect. Immun. 71 1689-1705 (2003)

74. Tokarz, R., Anderton, J. M., Katona, L. I. \& Benach, J. L. Combined effects of blood and temperature shift on Borrelia burgdorferi gene expression as determined by whole genome DNA array. Infect. Immun. 72, 5419-5432 (2004).

75. Revel, A. T. et al. bptA (bbe 16) is essential for the persistence of the Lyme disease spirochete, Borrelia burgdorferi, in its natural tick vector. Proc. Natl Acad. Sci. USA 102, 6972-6977 (2005).

76. Schwan, T. G., Piesman, J., Golde, W. T., Dolan, M. C. $\&$ Rosa, P. A. Induction of an outer surface protein on Borrelia burgdorferi during tick feeding. Proc. Natl Acad. Sci. USA 92, 2909-2913 (1995). The seminal demonstration of the induction of OspC during the nymphal blood meal and the importance of temperature as a stimulus for differential gene expression.

77. Piesman, J., Schneider, B. S. ¿ Zeidner, N. S. Use of quantitative PCR to measure density of Borrelia burgdorferi in the midgut and salivary glands of feeding tick vectors. J. Clin. Microbiol. 39, 4145-4148 (2001)

78. Coburn, J. \& Cugini, C. Targeted mutation of the outer membrane protein P66 disrupts attachment of the Lyme disease agent, Borrelia burgdorferi, to integrin $\alpha_{1} \beta_{3}$. Proc. Natl Acad. Sci. USA 100, 7301-7306 (2003) The first report to use targeted mutagenesis to show that a borrelial outer-membrane-spanning protein has a role in cytoadherence.

79. Pinne, M. et al. Elimination of channel-forming activity by insertional inactivation of the $p 66$ gene in Borrelia burgdorferi. FEMS Microbiol. Lett. 266, 241-249 (2007)

80. Norris, S. J., Coburn, J., Leong, J. M., Hu, L. T. \& Hook, M. in Borrelia: Molecular Biology, Host Interaction, and Pathogenesis (eds Samuels, D. S. \& Radolf, J. D.) 299-331 (Caister Academic, Norfolk, UK, 2010).
81. Hellwage, J. et al. The complement regulator factor $\mathrm{H}$ binds to the surface protein OspE of Borrelia burgdorferi. J. Biol. Chem. 276, 8427-8435 (2001).

82. Kenedy, M. R. \& Akins, D. R. The OspE-related proteins inhibit complement deposition and enhance serum resistance of Borrelia burgdorferi, the Lyme disease spirochete. Infect. Immun. 79, 1451-1457 (2011)

83. Stevenson, B., El-Hage, N., Hines, M. A., Miller, J. C. \& Babb, K. Differential binding of host complement inhibitor factor $\mathrm{H}$ by Borrelia burgdorferi Erp surface proteins: a possible mechanism underlying the expansive host range of Lyme disease spirochetes. Infect. Immun. 70, 491-497 (2002).

84. Kraiczy, P. et al. Complement resistance of Borrelia burgdorferi correlates with the expression of BbCRASP-1, a novel linear plasmid-encoded surface protein that interacts with human factor $\mathrm{H}$ and $\mathrm{FHL}-1$ and is unrelated to Erp proteins. J. Biol. Chem. 279 2421-2429 (2004)

85. Siegel, C. et al. Complement factor H-related proteins CFHR2 and CFHR5 represent novel ligands for the infection-associated CRASP proteins of Borrelia burgdorferi. PLoS ONE 5, e13519 (2010).

86. de Silva, A. M. \& Fikrig, E. Growth and migration of Borrelia burgdorferi in Ixodes ticks during blood feeding. Am. J. Trop. Med. Hyg. 53, 397-404 (1995) A detailed analysis of the route of spirochaete dissemination and the kinetics of spirochaete replication during the nymphal blood meal; this paper also provides additional evidence for salivary transmission of spirochaetes.

87. Ohnishi, J., Piesman, J. \& de Silva, A. M. Antigenic and genetic heterogeneity of Borrelia burgdorfer populations transmitted by ticks. Proc. Natl Acad. Sci. USA 98, 670-675 (2001).

A meticulous characterization of the expression profiles of OspA and OspC during tick dissemination.

88. Coleman, J. L. et al. Plasminogen is required for efficient dissemination of $B$. burgdorferi in ticks and for enhancement of spirochetemia in mice. Cell 89, 1111-1119 (1997)

A classic demonstration of how $B$. burgdorferi appropriates mammalian serum proteins to facilitate penetration of tick tissue barriers.

89. Zhang, L. et al. Molecular interactions that enable movement of the Lyme disease agent from the tick gut into the hemolymph. PLoS Pathog. 7, e1002079 (2011).

90. Coleman, J. L. et al. Borrelia burgdorferi binds plasminogen, resulting in enhanced penetration of endothelial monolayers. Infect. Immun. 63 2478-2484 (1995).

91. Guo, X. et al. Inhibition of neutrophil function by two tick salivary proteins. Infect. Immun. 77, 2320-2329 (2009).

92. Kotsyfakis, M. et al. Antiinflammatory and immunosuppressive activity of sialostatin L, a salivary cystatin from the tick Ixodes scapularis. J. Biol. Chem 281, 26298-26307 (2006)

93. Ramamoorthi, N. et al. The Lyme disease agen exploits a tick protein to infect the mammalian host. Nature 436, 573-577 (2005)

94. Pal, U. et al. OspC facilitates Borrelia burgdorferi invasion of Ixodes scapularis salivary glands. J. Clin. Invest. 113, 220-230 (2004).

95. Grimm, D. et al. Outer-surface protein C of the Lyme disease spirochete: a protein induced in ticks for infection of mammals. Proc. Natl Acad. Sci. USA 101 3142-3147 (2004)

A definitive demonstration that OspC is a borrelial virulence determinant following infection of mammals via needle or tick.

96. Tilly, K. et al. Borrelia burgdorferi OspC protein required exclusively in a crucial early stage of mammalian infection. Infect. Immun. 74, 3554-3564 (2006).

97. Petzke, M. M., Brooks, A., Krupna, M. A., Mordue, D \& Schwartz, I. Recognition of Borrelia burgdorferi, the Lyme disease spirochete, by TLR7 and TLR9 induces a type I IFN response by human immune cells. J. Immunol. 183, 5279-5292 (2009). The first report to show that borreliae activate host immune responses via endosomal TLRs.

98. Cervantes, J. L. et al. Phagosomal signaling by Borrelia burgdorferi in human monocytes involves Tolllike receptor (TLR) 2 and TLR8 cooperativity and TLR8-mediated induction of IFN- $\beta$. Proc. Natl Acad. Sci. USA 108, 3683-3688 (2011).

A paper confirming $B$. burgdorferi degradation in phagosomes as the principal mechanism for TLR signalling, and showing TLR2-TLR8 cooperativity. 
99. Miller, J. C. et al. A critical role for type I IFN in arthritis development following Borrelia burgdorfer infection of mice. J. Immunol. 181, 8492-8503 (2008).

A landmark paper calling attention to the involvement of type I IFNs in Lyme disease.

100. Shin, O. S. et al. Distinct roles for MyD88 and Toll-like receptors 2, 5, and 9 in phagocytosis of Borrelia burgdorferi and cytokine induction. Infect. Immun. 76 2341-2351 (2008)

101. Marre, M. L., Petnicki-Ocwieja, T., DeFrancesco, A. S Darcy, C. T. \& Hu, L. T. Human integrin $\alpha_{3} \beta_{1}$ regulates TLR2 recognition of lipopeptides from endosomal compartments. PLOS ONE 5, e12871 (2010).

102. Salazar, J. C. et al. Coevolution of markers of innate and adaptive immunity in skin and peripheral blood of patients with erythema migrans. J. Immunol. 171, 2660-2670 (2003).

103. Duray, P. H. Histopathology of clinical phases of human Lyme disease. Rheum. Dis. Clin. North Am. 15, 691-710 (1989).

104. Jones, K. L. et al. Higher mRNA levels of chemokines and cytokines associated with macrophage activation in erythema migrans skin lesions in patients from the United States than in patients from Austria with Lyme borreliosis. Clin. Infect. Dis. 46, 85-92 (2008).

105. Shih, C. M., Pollack, R. J., Telford, S. R. III \& Spielman, A. Delayed dissemination of Lyme disease spirochetes from the site of deposition in the skin of mice. J. Infect. Dis. 166, 827-831 (1992).

106. Brissette, C. A. et al. Borrelia burgdorferi infectionassociated surface proteins ErpP, ErpA, and ErpC bind human plasminogen. Infect. Immun. 77, 300-306 (2009).

107. Behera, A. K. et al. Borrelia burgdorferi BBB07 interaction with integrin $\alpha_{3} \beta$, stimulates production of pro-inflammatory mediators in primary human chondrocytes. Cell. Microbiol. 10, 320-331 (2008)

108. Guo, B. P., Norris, S. J., Rosenberg, L. C. \& Hook, M. Adherence of Borrelia burgdorferi to the proteoglycan decorin. Infect. Immun. 63, 3467-3472 (1995).

109. Parveen, N., Caimano, M., Radolf, J. D. \& Leong, J. M Adaptation of the Lyme disease spirochaete to the mammalian host environment results in enhanced glycosaminoglycan and host cell binding. $\mathrm{Mol}$. Microbiol. 47, 1433-1444 (2003).

110. Brissette, C. A., Verma, A., Bowman, A., Cooley, A. E. $\&$ Stevenson, B. The Borrelia burgdorferi outer-surface protein ErpX binds mammalian laminin. Microbiology 155, 863-872 (2009).

111. Verma, A., Brissette, C. A., Bowman, A. \& Stevenson, B. Borrelia burgdorferi BmpA is a laminin-binding protein. Infect. Immun. 77, 4940-4946 (2009).

112. Seshu, J. et al. Inactivation of the fibronectin-binding adhesin gene $b b k 32$ significantly attenuates the infectivity potential of Borrelia burgdorferi. Mol. Microbiol. 59, 1591-1601 (2006).

113. Brissette, C. A., Bykowski, T., Cooley, A. E., Bowman, A. \& Stevenson, B. Borrelia burgdorferi RevA antigen binds host fibronectin. Infect. Immun. 77, 2802-2812 (2009).

114. Olson, C. M. Jr et al. Local production of IFN- $\gamma$ by invariant NKT cells modulates acute Lyme carditis. J. Immunol. 182, 3728-3734 (2009).

115. Brown, C. R., Blaho, V. A., Fritsche, K. L. \& Loiacono, C. M. Stat 1 deficiency exacerbates carditis but not arthritis during experimental Lyme borreliosis. J. Interferon Cytokine Res. 26, 390-399 (2006)

116. Kinjo, Y. et al. Natural killer T cells recognize diacylglycerol antigens from pathogenic bacteria. Nature Immunol. 7, 978-986 (2006). A remarkable biochemical and immunological study showing that borrelial glycolipids activate natural killer T cells.

117. Lee, W. Y. et al. An intravascular immune response to Borrelia burgdorferi involves Kupffer cells and iNKT cells. Nature Immunol. 11, 295-302 (2010).

118. McKisic, M. D., Redmond, W. L. \& Barthold, S. W. Cutting edge: $T$ cell-mediated pathology in murine Lyme borreliosis. J. Immunol. 164, 6096-6099 (2000).

119. Connolly, S. E. \& Benach, J. L. The versatile roles of antibodies in Borrelia infections. Nature Rev. Microbiol. 3, 411-420 (2005)

120. Belperron, A. A., Dailey, C. M., Booth, C. J. \& Bockenstedt, L. K. Marginal zone B-cell depletion impairs murine host defense against Borrelia burgdorferi infection. Infect. Immun. 75, 3354-3360 (2007).
121. Tunev, S. S. et al. Lymphoadenopathy during Lyme borreliosis is caused by spirochete migration-induced specific B cell activation. PLoS Pathog. 7, e1002066 (2011).

122. Wooten, R. M. et al. Toll-like receptor 2 is required for innate, but not acquired, host defense to Borrelia burgdorferi. J. Immunol. 168, 348-355 (2002).

123. Bolz, D. D. et al. MyD88 plays a unique role in host defense but not arthritis development in Lyme disease. J. Immunol. 173, 2003-2010 (2004).

124. Liang, F. T., Jacobs, M. B., Bowers, L. C. \& Philipp, M. T. An immune evasion mechanism for spirochetal persistence in Lyme borreliosis. J. Exp. Med. 195, 415-422 (2002).

An elegant study demonstrating that OspC-specific antibodies select for spirochaetes that have downregulated expression of the lipoprotein during early mouse infection.

125. Zhang, J. R., Hardham, J. M., Barbour, A. G. \& Norris, $\mathrm{S}$. J. Antigenic variation in Lyme disease Borreliae by promiscuous recombination of VMP-like sequence cassettes. Cell 89, 275-285 (1997). The first report to describe the vls system for antigenic variation by $B$. burgdorferi.

126. Norris, S. J. Antigenic variation with a twist-the Borrelia story. Mol. Microbiol. 60, 1319-1322 (2006)

127. Petnicki-Ocwieja, T. et al. NOD2 suppresses Borrelia burgdorferi mediated murine Lyme arthritis and carditis through the induction of tolerance. PLOS ONE 6, e17414 (2011)

128. Oliver, J. H. Jr et al. An enzootic transmission cycle of Lyme borreliosis spirochetes in the southeastern United States. Proc. Natl Acad. Sci. USA 100 11642-11645 (2003).

129. Krupka, I. \& Straubinger, R. K. Lyme borreliosis in dogs and cats: background, diagnosis, treatment and prevention of infections with Borrelia burgdorferi sensu stricto. Vet. Clin. North Am. Small Anim. Pract. 40, 1103-1119 (2010)

130. Radolf, J. D., Bourell, K. W., Akins, D. R., Brusca, J. S. $£$ Norgard, M. V. Analysis of Borrelia burgdorferi membrane architecture by freeze-fracture electron microscopy. J. Bacteriol. 176, 21-31 (1994).

131. Rogers, E. A. et al. Rrp1, a cyclic-di-GMP-producing response regulator, is an important regulator of Borrelia burgdorferi core cellular functions. $\mathrm{Mol}$. Microbiol. 71, 1551-1573 (2009).

132. Pal, U. et al. A differential role for BB0365 in the persistence of Borrelia burgdorferi in mice and ticks. J. Infect. Dis. 197, 148-155 (2008)

133. Pal, U. et al. Borrelia burgdorferi basic membrane proteins $A$ and $B$ participate in the genesis of Lym arthritis. J. Exp. Med. 205, 133-141 (2008).

134. Boylan, J. A. et al. Borrelia burgdorferi bb0728 encodes a coenzyme A disulphide reductase whose function suggests a role in intracellular redox and the oxidative stress response. Mol. Microbiol. 59 475-486 (2006)

135. Eggers, C. H. et al. The coenzyme A disulfide reductase of Borrelia burgdorferi is important for rapid growth throughout the enzootic cycle and essential for infection of the mammalian host. Mol. Microbiol. 82 679-697 (2011).

136. Freedman, J. C. et al. Identification and molecular characterization of a cyclic-di-GMP effector protein, PlzA (BB0733): additional evidence for the existence of a functional cyclic-di-GMP regulatory network in the Lyme disease spirochete, Borrelia burgdorferi. FEMS Immunol. Med. Microbiol. 58, 285-294 (2010).

137. Pitzer, J. E. et al. Analysis of the Borrelia burgdorferi cyclic-di-GMP-binding protein PIzA reveals a role in motility and virulence. Infect. Immun. 79, 1815-1825 (2011).

138. Salman-Dilgimen, A., Hardy, P. O., Dresser, A. R. \& Chaconas, G. HrpA, a DEAH-box RNA helicase, is involved in global gene regulation in the Lyme disease spirochete. PLOS ONE 6, e22168 (2011)

139. Chaconas, G., Stewart, P. E., Tilly, K., Bono, J. L. \& Rosa, P. Telomere resolution in the Lyme disease spirochete. EMBO J. 20, 3229-3237 (2001). An extraordinary demonstration that cp26-encoded ResT is required for telomere resolution during replication of the borrelial linear chromosome and plasmids.

140. Jewett, M. W. et al. GuaA and GuaB are essential for Borrelia burgdorferi survival in the tick-mouse infection cycle. J. Bacteriol. 191, 6231-6241 (2009).
141 Metts, M. S., McDowell, J V., Theisen, M., Hansen, P. R. \& Marconi, R. T. Analysis of the OspE determinants involved in binding of factor $\mathrm{H}$ and OspE-targeting antibodies elicited during Borrelia burgdorferi infection in mice. Infect. Immun. 71 3587-3596 (2003).

142. Kraiczy, P. et al. Immune evasion of Borrelia burgdorferi: mapping of a complement-inhibitor facto $\mathrm{H}$-binding site of BbCRASP-3, a novel member of the Erp protein family. Eur. J. Immunol. 33, 697-707 (2003).

143. Kawabata, H., Norris, S. J. \& Watanabe, H. BBE02 disruption mutants of Borrelia burgdorferi B31 have a highly transformable, infectious phenotype. Infect. Immun. 72, 7147-7154 (2004).

144. Purser, J. E. et al. A plasmid-encoded nicotinamidase (PncA) is essential for infectivity of Borrelia burgdorferi in a mammalian host. Mol. Microbiol. 48 753-764 (2003)

145. Hartmann, K. et al. Functional characterization of BbCRASP-2, a distinct outer membrane protein of Borrelia burgdorferi that binds host complement regulators factor $\mathrm{H}$ and FHL-1. Mol Microbiol. 61 1220-1236 (2006).

146. Jewett, M. W. et al. The critical role of the linear plasmid Ip36 in the infectious cycle of Borrelia burgdorferi. Mol. Microbiol. 64, 1358-1374 (2007).

147. Fischer, J. R., LeBlanc, K. T. \& Leong, J. M. Fibronectin binding protein BBK32 of the Lyme disease spirochete promotes bacterial attachment to glycosaminoglycans. Infect. Immun. 74, 435-441 (2006).

148. Blevins, J. S., Hagman, K. E. \& Norgard, M. V. Assessment of decorin-binding protein A to the infectivity of Borrelia burgdorferi in the murine models of needle and tick infection. BMC Microbiol. 8, 82 (2008).

149. Brown, E. L. et al. Resistance to Lyme disease in decorin-deficient mice. J. Clin. Invest. 107, 845-852 (2001).

150. Shi, Y., Xu, Q., McShan, K. \& Liang, F. T. Both decorinbinding proteins $A$ and $B$ are critical for the overall virulence of Borrelia burgdorferi. Infect. Immun. 76 , 1239-1246 (2008).

151. Kumar, M., Yang, X., Coleman, A. S. \& Pal, U. BBA52 facilitates Borrelia burgdorferi transmission from feeding ticks to murine hosts. J. Infect. Dis. 201, 1084-1095 (2010).

152. Promnares, K. et al. Borrelia burgdorferi small lipoprotein Lp6.6 is a member of multiple protein complexes in the outer membrane and facilitates pathogen transmission from ticks to mice. Mol. Microbiol. 74, 112-125 (2009).

153. Gilmore, R. D. Jr et al. The bba64 gene of Borrelia burgdorferi, the Lyme disease agent, is critical for mammalian infection via tick bite transmission. Proc. Natl Acad. Sci. USA 107, 7515-7520 (2010).

154. Bykowski, T. et al. Coordinated expression of Borrelia burgdorferi complement regulator-acquiring surface proteins during the Lyme disease spirochete's mammal-tick infection cycle. Infect. Immun. 75 4227-4236 (2007)

155. McDowell, J. V. et al. Evidence that the BBA68 protein (BbCRASP-1) of the Lyme disease spirochetes does not contribute to factor $\mathrm{H}$-mediated immune evasion in humans and other animals. Infect. Immun. 74, 3030-3034 (2006).

\section{Acknowledgements}

The authors gratefully acknowledge support from the US National Institutes of Health, National Institute for Allergy and Infectious Diseases (grants Al029735 and AI029735-20S1 to J.D.R. and M.J.C.; AI085248 to M.J.C. AI044254 to B.S.; and AI071107, Al068799, AI082436 and Al080646 to L.T.H.). The authors also thank S. Dunham-Ems, T. Petnicki-Ocwieja, E. Troy and C. Brissette for invaluable assistance with the figures and the table, many insightful comments and careful proofreading.

Competing interests statement

The authors declare no competing financial interests.

\section{FURTHER INFORMATION}

Justin D. Radolf's homepage:

http://spirocheteresearch.uchc.edu/

ALL LINKS ARE ACTIVE IN THE ONLINE PDF 